\title{
ISOMORPHISMS BETWEEN LEAVITT ALGEBRAS AND THEIR MATRIX RINGS
}

\author{
G. ABRAMS, P. N. ÁNH, AND E. PARDO
}

\begin{abstract}
Let $K$ be any field, let $L_{n}$ denote the Leavitt algebra of type $(1, n-1)$ having coefficients in $K$, and let $\mathrm{M}_{d}\left(L_{n}\right)$ denote the ring of $d \times d$ matrices over $L_{n}$. In our main result, we show that $\mathrm{M}_{d}\left(L_{n}\right) \cong L_{n}$ if and only if $d$ and $n-1$ are coprime. We use this isomorphism to answer a question posed in 14 regarding isomorphisms between various $\mathrm{C}^{*}$ algebras. Furthermore, our result demonstrates that data about the $K_{0}$ structure is sufficient to distinguish up to isomorphism the algebras in an important class of purely infinite simple $K$-algebras.
\end{abstract}

\section{INTRODUCTION}

Let $K$ be any field, and let $m<n$ be positive integers. The ring $R$ is said to have invariant basis number (IBN) if no two free left $R$-modules of differing rank over $R$ are isomorphic. On the other hand, $R$ is said to have module type $(m, n-m)$ in case for every pair of positive integers $a$ and $b,(1)$ if $1 \leq a<m$ then the free left $R$-modules $R^{a}$ and $R^{i}$ are not isomorphic for all positive integers $i \neq a$, and (2) if $a, b \geq m$, then the free left $R$-modules $R^{a}$ and $R^{b}$ are isomorphic precisely when $a \equiv b(\bmod n-m)$. It is not hard to show that any non-IBN ring has module type $(m, n-m)$ for some pair of positive integers $m<n$. (The notation used here is not completely universal: some authors refer to the module type of such an algebra as the pair $(m, n)$. Our notation is consistent with that used in many of the algebra articles on this topic, and is also consistent with the $\mathrm{C}^{*}$-algebra usage as well.) As shown by Leavitt in [12, for every such pair $m, n$ there exists a $K$-algebra $L_{K}(m, n)$ whose module type is $(m, n-m)$. In particular, the module type of $L_{K}(1, n)$ is $(1, n-1)$. We denote $L_{K}(1, n)$ by $L_{n}$. Various aspects of these algebras have been investigated, with an initial flurry of activity in the 1960's and early 1970's (e.g. [7], [8], and [13]), and then again in a revival beginning at the start of the new millennium (e.g. [1], 2], and [6]).

Date: 18 December 2006.

2000 Mathematics Subject Classification. Primary 16D70, Secondary 46L05.

Key words and phrases. Leavitt algebra, Cuntz algebra.

The first author is grateful for support provided by the Clear Creek Ranch Institute and its directors, L. George and S. Hyde.

The second author is supported partly by Hungarian National Foundation for Scientific Research grant no. K61007. During Fall 2006 he was also supported partly by The Colorado College, the University of Colorado at Colorado Springs, and Professor Michael Siddoway.

The third author was partially supported by the DGI and European Regional Development Fund, jointly, through Project MTM2004-00149, by PAI III projects FQM-298 and P06-FQM-1889 of the Junta de Andalucía, by the Comissionat per Universitats i Recerca de la Generalitat de Catalunya, and by the Consolider Ingenio "Mathematica" project CSD2006-32 by the MEC. 
On the "analytic" side of the coin, Cuntz [9] in the 1970s investigated the C*-algebras $\left\{\mathcal{O}_{n} \mid 2 \leq n \in \mathbb{N}\right\}$. There is an intimate connection between the Leavitt algebra $L_{K}(1, n)$ and the Cuntz algebra $\mathcal{O}_{n}$. Specifically, for any field $K$, the elements of $L_{K}(1, n)$ can be viewed as linear transformations on an infinite dimensional $K$-vector space in a natural way as a collection of shift operators. In particular, when $K$ is the field of complex numbers, then $L_{K}(1, n)$ can be viewed as acting on Hilbert space $\ell^{2}$, and thereby inherits the operator norm. The Cuntz algebra $\mathcal{O}_{n}$ is the completion of $L_{\mathbb{C}}(1, n)$ in the metric induced by this norm.

Since $L_{n} \cong L_{n}^{n}$ as free left $L_{n}$-modules, by taking endomorphism rings we get immediately that there is a ring isomorphism between $L_{n}$ and $\mathrm{M}_{n}\left(L_{n}\right)$. The first two authors extended this type of isomorphism to additional matrix sizes in [2], where they observe that $L_{n} \cong$ $\mathrm{M}_{d}\left(L_{n}\right)$ whenever $d$ divides $n^{\alpha}$ for some positive integer $\alpha$. In [12] Leavitt shows that for $\operatorname{gcd}(d, n-1)>1$, the $K$-algebras $L_{n}$ and $\mathrm{M}_{d}\left(L_{n}\right)$ cannot be isomorphic. Since $d \mid n^{\alpha}$ implies $\operatorname{gcd}(d, n-1)=1$, these two results yield the following natural question, posed in [2], page 362:

For $\operatorname{gcd}(d, n-1)=1$, are $L_{n}$ and $\mathrm{M}_{d}\left(L_{n}\right)$ isomorphic?

In our main result, Theorem 4.14, we answer this question in the affirmative for all fields $K$.

Theorem 4.14 has important consequences in the context of $\mathrm{C}^{*}$-algebras. First, we show in Section 5 that this result can be used to directly answer in the affirmative the following question, posed in [14, page 8:

Are $\mathrm{M}_{m}\left(\mathcal{O}_{n}\right)$ and $\mathcal{O}_{n}$ isomorphic whenever $m$ and $n-1$ are relatively prime?

While an affirmative answer to this question was provided for even $n$ in [18], Corollary 7.3, and subsequently shown for all $n \geq 2$ as a consequence of [15], Theorem 4.3(1), the method we provide here is significantly more elementary. Indeed, the second important consequence of our result is that, unlike the current situation in the $\mathrm{C}^{*}$-algebra case, the isomorphisms we present between the indicated $K$-algebras are in fact explicitly given. Moreover, when $K=\mathbb{C}$, this explicit description carries over to an explicit description of the isomorphisms between the appropriately sized matrix rings over Cuntz algebras.

Finally, our result demonstrates that data about the $K_{0}$ structure is sufficient to distinguish up to isomorphism the algebras in an important class of purely infinite simple $K$-algebras, thus paving a path for subsequent work by the authors [3] towards an algebraic version of [16], Theorem 4.2.4.

The authors thank the referee for an extremely careful review of this article.

\section{Notation AND BASiC CONCEPTS}

We begin by explicitly defining the Leavitt algebras $L_{K}(1, n)$. For any positive integer $n \geq 2$, and field $K$, we denote $L_{K}(1, n)$ by $L_{K, n}$, and call it the Leavitt algebra of type $(1, n-1)$ with coefficients in $K$. (When $K$ is understood, we denote this algebra simply by $L_{n}$.) Precisely, $L_{K, n}$ is the quotient of the free associative $K$-algebra in $2 n$ variables:

$$
L_{K, n}=K<X_{1}, \ldots, X_{n}, Y_{1}, \ldots, Y_{n}>/ T
$$

where $T$ is the ideal generated by the relations $X_{i} Y_{j}-\delta_{i j} 1_{K}($ for $1 \leq i, j \leq n)$ and $\sum_{j=1}^{n} Y_{j} X_{j}-$ $1_{K}$. The images of $X_{i}, Y_{i}$ in $L_{K, n}$ are denoted respectively by $x_{i}, y_{i}$. In particular, we have the equalities $x_{i} y_{j}=\delta_{i j} 1_{K}$ and $\sum_{j=1}^{n} y_{j} x_{j}=1_{K}$ in $L_{n}$. The algebra $L_{n}$ was investigated 
originally by Leavitt in his seminal paper [12]. We now list various fundamental properties of $L_{n}$, culminating in the property which will serve as the focus of our investigation.

Proposition 1.1. Let $K$ be any field.

(1) [12, Theorem 8: $L_{n}$ has module type $(1, n-1)$. In particular, if $a \equiv b(\bmod n-1)$ then $L_{n}^{a} \cong L_{n}^{b}$ as free left $L_{n}$-modules. Consequently, if $a \equiv b(\bmod n-1)$, then there is an isomorphism of matrix rings $\mathrm{M}_{a}\left(L_{n}\right) \cong \mathrm{M}_{b}\left(L_{n}\right)$.

(2) Suppose $R$ is a $K$-algebra which contains a subset $\left\{a_{1}, \ldots, a_{n}, b_{1}, \ldots, b_{n}\right\}$ for which $a_{i} b_{j}=$ $\delta_{i j} 1_{R}($ for $1 \leq i, j \leq n)$, and $\sum_{j=1}^{n} b_{j} a_{j}=1_{R}$. (For instance, any $K$-algebra having module type $(1, n-1)$ has this property.) Then there exists a (unital) $K$-algebra homomorphism from $L_{n}$ to $R$ extending the $\operatorname{map} x_{i} \mapsto a_{i}$ and $y_{i} \mapsto b_{i}($ for $1 \leq i \leq n)$.

(3) [13], Theorem 2: $L_{n}$ is a simple $K$-algebra.

Corollary 1.2. Let I denote the identity matrix in $\mathrm{M}_{d}\left(L_{n}\right)$. To show $L_{n} \cong \mathrm{M}_{d}\left(L_{n}\right)$ it suffices to show that there is a set $S=\left\{a_{1}, \ldots, a_{n}, b_{1}, \ldots, b_{n}\right\} \subseteq \mathrm{M}_{d}\left(L_{n}\right)$ such that: $a_{i} b_{j}=\delta_{i j} I$ (for $1 \leq i, j \leq n) ; \sum_{j=1}^{n} b_{j} a_{j}=I$; and $S$ generates $\mathrm{M}_{d}\left(L_{n}\right)$ as a $K$-algebra.

Proof. The existence of a nontrivial $K$-algebra homomorphism from $L_{n}$ to $\mathrm{M}_{d}\left(L_{n}\right)$ follows from Proposition 1.1(2), while the injectivity of such a homomorphism follows from Proposition 1.1(3). Since $\left\{x_{1}, \ldots, x_{n}, y_{1}, \ldots, y_{n}\right\}$ generates $L_{n}$ as a $K$-algebra, the image of this homomorphism is generated by $\left\{a_{1}, \ldots, a_{n}, b_{1}, \ldots, b_{n}\right\} \subseteq \mathrm{M}_{d}\left(L_{n}\right)$.

For any unital $\operatorname{ring} R$ and $i \in\{1,2, \ldots, d\}$ we denote the idempotent $e_{i, i}$ of the matrix ring $\mathrm{M}_{d}(R)$ simply by $e_{i}$, and we define

$$
E_{i}=\sum_{j=1}^{i} e_{j} .
$$

In this notation $E_{d}=I$, the identity matrix in $\mathrm{M}_{d}(R)$.

Definition 1.3. For any field $K$, the extension of the assignments $x_{i} \mapsto y_{i}=x_{i}^{*}$ and $y_{i} \mapsto$ $x_{i}=y_{i}^{*}$ for $1 \leq i \leq n$ yields an involution $*$ on $L_{K}(1, n)$. This involution on $L_{K}(1, n)$ produces an involution on any sized matrix ring $\mathrm{M}_{m}\left(L_{K}(1, n)\right)$ over $L_{K}(1, n)$ by setting $X^{*}=\left(x_{j, i}^{*}\right)$ for each $X=\left(x_{i, j}\right) \in \mathrm{M}_{m}\left(L_{K}(1, n)\right)$.

We note that if $K$ is a field with involution (which we also denote by $*$ ), then a second involution on $L_{K}(1, n)$ may be defined by extending the assignments $k \mapsto k^{*}$ for all $k \in K$, $x_{i} \mapsto y_{i}=x_{i}^{*}$ and $y_{i} \mapsto x_{i}=y_{i}^{*}$ for $1 \leq i \leq n$. Of course in the case $K=\mathbb{C}$ we have such an involution on $K$. Although it might be of interest to consider this second type of involution on $L_{\mathbb{C}}(1, n)$ in order to maintain some natural connection with the standard involution on the corresponding Cuntz algebra $\mathcal{O}_{n}$, we prefer to work with the involution on $L_{K}(1, n)$ described in Definition 1.3 because it can be defined for any field $K$. All of the results presented in this article for involutions on $L_{K}(1, n)$ and their matrix rings are valid using either type of involution.

We now set some notation which will be used throughout the remainder of the article. For positive integers $d$ and $n$ we write

$$
n=q d+r \text { where } 1 \leq r \leq d .
$$


We assume throughout that $\operatorname{gcd}(d, n-1)=1$, and that $d<n$. (We will relax the hypothesis $d<n$ in our main result.) Without loss of generality we will also assume that $r \geq 2$, since $r=1$ would yield $n-1=q d$, which along with the hypothesis that $\operatorname{gcd}(d, n-1)=1$ would yield $d=1$, and the main result in this case is then the trivial statement $L_{n} \cong \mathrm{M}_{1}\left(L_{n}\right)$. An important role will be played by the number $s$, defined as

$$
s=d-(r-1) \text {. }
$$

Since $\operatorname{gcd}(d, n-1)=1$ we get also that $\operatorname{gcd}(s, d)=1$.

Definition 1.4. We consider the sequence $\left\{h_{i}\right\}_{i=1}^{d}$ of integers, whose $i^{\text {th }}$ entry is given by

$$
h_{i}=1+(i-1) s(\bmod d) .
$$

The integers $h_{i}$ are understood to be taken from the set $\{1,2, \ldots, d\}$. Rephrased, we define the sequence $\left\{h_{i}\right\}_{i=1}^{d}$ by setting $h_{1}=1$, and, for $1 \leq i \leq d-1$,

$$
h_{i+1}=h_{i}+s \text { if } h_{i} \leq r-1, \quad \text { and } \quad h_{i+1}=h_{i}-(r-1) \text { if } h_{i} \geq r .
$$

Because $\operatorname{gcd}(d, s)=1$ (so that $s$ is invertible mod $d$ ), basic number theory yields the following

\section{Lemma 1.5.}

(1) The entries in the sequence $h_{1}, h_{2}, \ldots, h_{d}$ are distinct.

(2) The set of entries $\left\{h_{1}, h_{2}, \ldots, h_{d}\right\}$ equals the set $\{1,2, \ldots, d\}$ (in some order).

(3) The final entry in the sequence is $r$; that is, $h_{d}=r$.

Proof. The only non-standard statement is (3). Suppose $r=1+(i-1) s(\bmod d)$. Then $r-1=(i-1) s(\bmod d)$, so that $(r-1)+s=i s(\bmod d)$. But $d=(r-1)+s$ by definition, so this gives $d=i s(\bmod d)$. Now $\operatorname{gcd}(s, d)=1$ gives that $i=d$, so that $i-1=d-1$ and we get $r=1+(d-1) s(\bmod d)=h_{d}$ as desired.

Our interest will lie in a decomposition of $\{1,2, \ldots, d\}$ effected by the sequence $h_{1}, h_{2}, \ldots, h_{d}$, as follows.

Definition 1.6. We let $d_{1}$ denote the integer for which

$$
h_{d_{1}}=r-1
$$

in the previously defined sequence. Such an integer $d_{1}$ exists by Lemma 1.5)(2). Note then that $h_{d_{1}+1}=(r-1)+s=d$. We denote by $\hat{S}_{1}$ the following subset of $\{1,2, \ldots, d\}$ :

$$
\hat{S}_{1}=\left\{h_{i} \mid 1 \leq i \leq d_{1}\right\} \text {. }
$$

We denote by $\hat{S}_{2}$ the complement of $\hat{S}_{1}$ in $\{1,2, \ldots, d\}$; in other words, $\hat{S}_{2}=\left\{h_{i} \mid d_{1}+1 \leq i \leq d\right\}$. If we define $d_{2}=d-d_{1}$, then

$$
d_{1}=\left|\hat{S}_{1}\right|, d_{2}=\left|\hat{S}_{2}\right|, \text { and } d_{1}+d_{2}=d .
$$

Let $e_{1}=\left|\hat{S}_{1} \cap\{r-1, r, r+1, \ldots d\}\right|$. So $e_{1}$ is the number of elements in $\hat{S}_{1}$ which are at least $r-1$. Similarly, let $e_{2}=\left|\hat{S}_{2} \cap\{r-1, r, r+1, \ldots d\}\right|$. (Note by definition of $\hat{S}_{1}$ and Lemma 1.5(3) we have $1, r-1 \in \hat{S}_{1}$ and $r, d \in \hat{S}_{2}$.) So we get

$$
e_{1}+e_{2}=|\{r-1, r, \ldots, d\}|=d-(r-1)+1=d-r+2 .
$$


Let $f_{1}=\left|\hat{S}_{1} \cap\{1,2, \ldots, r-1, r\}\right|$. So $f_{1}$ is the number of elements in $\hat{S}_{1}$ which are at most $r$. Similarly, let $f_{2}=\left|\hat{S}_{2} \cap\{1,2, \ldots, r\}\right|$. We get

$$
f_{1}+f_{2}=r
$$

Finally, by definition we have

$$
e_{1}+f_{1}=d_{1}+1 \text { and } e_{2}+f_{2}=d_{2}+1 \text {. }
$$

Proposition 1.7. Write $h_{d_{1}}=r-1=1+\left(d_{1}-1\right) s(\bmod d)$. So there exists a nonnegative integer $t$ with $r-1=1+\left(d_{1}-1\right) s-t d$, so that

$$
r-1=1+\left(d_{1}-1\right) s-t[s+(r-1)]=1+\left(d_{1}-1-t\right) s-t(r-1) .
$$

Let $b$ denote $d_{1}-1-t$. So we have

$$
r-1=1+b s-t(r-1) .
$$

(In particular, we also have $(1+t)(r-1)=1+b s$.) Then $e_{1}=t+1, d_{1}=1+b+t$, and $f_{1}=1+b$.

Proof. By definition, each element of the sequence $\left\{h_{i}\right\}_{i=1}^{d}$ is the remainder of $1+(i-1) s$ modulo $d$. Now, we will show by induction on $i$ that $h_{i}=1+(i-1) s-l_{i} d$ where $l_{i}$ is the number of $h_{j}$ for which $h_{j} \geq r$ and $j<i$.

For $i=1, h_{1}=1=1+(1-1) s-0 d$, as $r \geq 2$ implies $l_{1}=0$. Now, suppose that the result holds for $i \geq 1$. If $h_{i} \geq r, l_{i+1}=l_{i}+1$ by definition. Also, the computation gives us

$h_{i+1}=h_{i}-(r-1)=h_{i}+s-d=1+(i-1) s-l_{i} d+s-d=1+i s-\left(l_{i}+1\right) d=1+i s-l_{i+1} d$.

On the other hand, if $h_{i} \leq r-1$, then $l_{i+1}=l_{i}$ by definition. Also, the computation gives us

$$
h_{i+1}=h_{i}+s=1+(i-1) s-l_{i} d+s=1+i s-l_{i} d=1+i s-l_{i+1} d .
$$

Thus, induction step works.

Now, for $i=d_{1}$, denote $l_{d_{1}}$ by $t$. The previous assertion shows that

$$
r-1=1+\left(d_{1}-1\right) s-t d,
$$

where $t$ is the number of $h_{j}$ for which $h_{j} \geq r$ and $j<d_{1}$. Since $\hat{S}_{1}=\left\{h_{i} \mid 1 \leq i \leq d_{1}\right\}$, we have

$$
t=\left|\left(\hat{S}_{1} \backslash\{r-1\}\right) \cap\{r-1, r, \ldots, d\}\right|,
$$

so that $e_{1}=1+t$.

By definition of $b, d_{1}=1+b+t$. But $f_{1}=d_{1}+1-e_{1}$, so we are done.

Example 1.8. It will be helpful to give a specific example in order to solidify these ideas. Suppose $n=35, d=13$. Then $\operatorname{gcd}(13,35-1)=1$, so we are in the desired situation. Now $35=2 \cdot 13+9$, so that $r=9, r-1=8$, and $s=d-(r-1)=13-8=5$. Then the sequence $h_{1}, h_{2}, \ldots, h_{d}$ is given by

$$
1,6,11,3,8,13,5,10,2,7,12,4,9 \text {. }
$$

Since $r-1=8$, The partition $\{1,2, \ldots, d\}=\hat{S}_{1} \cup \hat{S}_{2}$ is then

$$
\{1,2, \ldots, 13\}=\{1,3,6,8,11\} \cup\{2,4,5,7,9,10,12,13\} .
$$

Furthermore,

$$
d_{1}=|\{1,3,6,8,11\}|=5, \quad d_{2}=|\{2,4,5,7,9,10,12,13\}|=8,
$$




$$
\begin{gathered}
e_{1}=|\{8,11\}|=2, \quad e_{2}=|\{9,10,12,13\}|=4, \\
f_{1}=|\{1,3,6,8\}|=4, \quad f_{2}=|\{2,4,5,7,9\}|=5 .
\end{gathered}
$$

Note that $f_{1}=4=1+3=1+b$, and $e_{1}=2=1+1=1+t$. Finally, we have

$$
r-1=8=1+3 \cdot 5-1 \cdot 8=1+b s-t(r-1) \text {. }
$$

\section{The SEARCH For APPROpRIATE MATRICES INSIDE $\mathrm{M}_{d}\left(L_{n}\right)$}

We start this section by giving a plausibility argument for Theorem 4.14. In [12], Theorem 5, Leavitt proves

Proposition 2.1. If $R$ has module type $(1, n-1)$, then $\mathrm{M}_{d}(R)$ has module type $\left(1, \frac{n-1}{\operatorname{gcd}(d, n-1)}\right)$.

Since module type is an isomorphism invariant, this result immediately gives that $L_{n}$ and $\mathrm{M}_{d}\left(L_{n}\right)$ are not isomorphic when $\operatorname{gcd}(d, n-1)>1$.

On the other hand, in case $\operatorname{gcd}(d, n-1)=1$, Leavitt's proof of Proposition 2.1 gives an algorithm for finding specific elements $\left\{a_{1}, \ldots, a_{n}, b_{1}, \ldots, b_{n}\right\}$ inside $\mathrm{M}_{d}\left(L_{n}\right)$ which satisfy the appropriate relations. So, by Corollary [1.2, we would be done if we could show that this set of elements generates $\mathrm{M}_{d}\left(L_{n}\right)$ as a $K$-algebra.

However, this set of elements does NOT generate $\mathrm{M}_{d}\left(L_{n}\right)$ in general. It is instructive here to look at a specific example. Because by [2], Proposition 2.1, we know our main result is true when $d$ divides some power of $n$, the smallest case of interest is the situation $d=3, n=5$, since then $\operatorname{gcd}(d, n-1)=1$ but $d$ does not divide any power of $n$. Leavitt's proof (for general $d, n)$ manifests in this specific case that $M_{3}\left(L_{5}\right)$ has module type $(1,4)$, and is based on an analysis of the $n=5$ elements in $M_{3}\left(L_{5}\right)$

$$
\begin{gathered}
X_{1}=\left(\begin{array}{lll}
x_{1} & 0 & 0 \\
x_{2} & 0 & 0 \\
x_{3} & 0 & 0
\end{array}\right) \quad X_{2}=\left(\begin{array}{ccc}
x_{4} & 0 & 0 \\
x_{5} & 0 & 0 \\
0 & x_{1} & 0
\end{array}\right) \quad X_{3}=\left(\begin{array}{lll}
0 & x_{2} & 0 \\
0 & x_{3} & 0 \\
0 & x_{4} & 0
\end{array}\right) \\
X_{4}=\left(\begin{array}{ccc}
0 & x_{5} & 0 \\
0 & 0 & x_{1} \\
0 & 0 & x_{2}
\end{array}\right) \quad X_{5}=\left(\begin{array}{lll}
0 & 0 & x_{3} \\
0 & 0 & x_{4} \\
0 & 0 & x_{5}
\end{array}\right)
\end{gathered}
$$

together with the five dual matrices $Y_{i}=X_{i}^{*}$ for $1 \leq i \leq 5$. While these ten matrices generate "much of" $M_{3}\left(L_{5}\right)$, these matrices do not, for instance, generate the matrix unit $e_{1,3}$. In fact, we show below in Proposition 6.3 that whenever $\operatorname{gcd}(d, n-1)=1$ but $d$ does not divide $n^{\alpha}$ for any positive integer $\alpha$, then the matrices in $\mathrm{M}_{d}\left(L_{n}\right)$ which arise in the proof of [12], Theorem 5, cannot generate $\mathrm{M}_{d}\left(L_{n}\right)$.

A breakthrough in this investigation was achieved when the authors were able to show that isomorphisms between more general structures (so-called "Leavitt path algebras"; see e.g. [4]), when interpreted in light of [5], Proposition 13, in fact yield an isomorphism between $L_{5}$ and $\mathrm{M}_{3}\left(L_{5}\right)$. By tracing through the appropriate translation maps, the following subset of $\mathrm{M}_{3}\left(L_{5}\right)$ emerges as the desired set of elements, elements which satisfy the appropriate relations and generate $\mathrm{M}_{3}\left(L_{5}\right)$ as a $K$-algebra:

$$
X_{1}=\left(\begin{array}{ccc}
x_{1} & 0 & 0 \\
x_{5} & 0 & 0 \\
x_{3} & 0 & 0
\end{array}\right) \quad X_{2}=\left(\begin{array}{ccc}
x_{2} & 0 & 0 \\
x_{4} & 0 & 0 \\
0 & 1 & 0
\end{array}\right) \quad X_{3}=\left(\begin{array}{ccc}
0 & 0 & x_{1}{ }^{2} \\
0 & 0 & x_{5} x_{1} \\
0 & 0 & x_{3} x_{1}
\end{array}\right)
$$




$$
X_{4}=\left(\begin{array}{ccc}
0 & 0 & x_{2} x_{1} \\
0 & 0 & x_{4} x_{1} \\
0 & 0 & x_{5}
\end{array}\right) \quad X_{5}=\left(\begin{array}{ccc}
0 & 0 & x_{2} \\
0 & 0 & x_{4} \\
0 & 0 & x_{3}
\end{array}\right)
$$

and $Y_{i}=X_{i}^{*}$ for each $1 \leq i \leq 5$. What we glean from this particular set of matrices in $\mathrm{M}_{3}\left(L_{5}\right)$ is that:

(i) it might be useful to use $1_{K}$ as an entry (any number of times) in the generating matrices,

(ii) various nonlinear monomials might play a useful role in the generating matrices, and

(iii) it might be of use to place elements in the matrices in some order other than lexicographic order.

With guidance provided by the above system of generators in $\mathrm{M}_{3}\left(L_{5}\right)$, one can easily check that the following set of matrices (together with the appropriate dual matrices) is also a set of generators of $\mathrm{M}_{3}\left(L_{5}\right)$ which satisfies the conditions of Corollary [1.2, and hence provides an isomorphism between $L_{5}$ and $\mathrm{M}_{3}\left(L_{5}\right)$.

$$
\begin{gathered}
X_{1}=\left(\begin{array}{lll}
x_{1} & 0 & 0 \\
x_{2} & 0 & 0 \\
x_{3} & 0 & 0
\end{array}\right) \quad X_{2}=\left(\begin{array}{ccc}
x_{4} & 0 & 0 \\
x_{5} & 0 & 0 \\
0 & 1 & 0
\end{array}\right) \\
X_{3}=\left(\begin{array}{ccc}
0 & 0 & x_{1}{ }^{2} \\
0 & 0 & x_{2} x_{1} \\
0 & 0 & x_{3} x_{1}
\end{array}\right) \quad X_{4}=\left(\begin{array}{ccc}
0 & 0 & x_{4} x_{1} \\
0 & 0 & x_{5} x_{1} \\
0 & 0 & x_{2}
\end{array}\right) \quad X_{5}=\left(\begin{array}{lll}
0 & 0 & x_{4} \\
0 & 0 & x_{5} \\
0 & 0 & x_{3}
\end{array}\right)
\end{gathered}
$$

It is easy to show, and not at all unexpected, that for each $n$, the symmetric group $S_{n}$ acts as automorphisms on $L_{n}$ in the obvious way. Specifically, for $\sigma \in S_{n}$ we define $\alpha_{\sigma}: L_{n} \rightarrow L_{n}$ by setting $\alpha_{\sigma}\left(x_{i}\right)=x_{\sigma(i)}$ for each $1 \leq i \leq n$, and extending linearly. In fact, with $\sigma \in S_{5}$ given by $\sigma(2)=5, \sigma(4)=2$, and $\sigma(5)=4$, it is straightforward to show that the corresponding $\alpha_{\sigma}$ transforms this last set of five matrices to the previously given set.

We close this section by giving three additional sets of generating matrices for $\mathrm{M}_{3}\left(L_{5}\right)$. First, consider the set $\left\{X_{1}, X_{2}, X_{3}, X_{4}, X_{5}\right\}$ of matrices presented directly above. It is relatively easy to show that by defining $X_{5}^{\prime}$ to be the matrix gotten by interchanging the entries $x_{5}$ and $x_{3}$ of $X_{5}$, then the set $\left\{X_{1}, X_{2}, X_{3}, X_{4}, X_{5}^{\prime}\right\}$ (and their duals) provide a generating set for $\mathrm{M}_{3}\left(L_{5}\right)$. (We note for future reference that, in contrast, switching the entries $x_{5}$ and $x_{4}$ of $X_{5}$ would not provide a generating set.)

Second, consider again the set $\left\{X_{1}, X_{2}, X_{3}, X_{4}, X_{5}\right\}$ of matrices presented directly above. It is not difficult to show that by defining $X_{4}^{\prime \prime}$ and $X_{5}^{\prime \prime}$ to be the matrices gotten by interchanging the entry $x_{2}$ of $X_{4}$ with the entry $x_{3}$ of $X_{5}$, then the set $\left\{X_{1}, X_{2}, X_{3}, X_{4}^{\prime \prime}, X_{5}^{\prime \prime}\right\}$ (and their duals) provide a generating set for $\mathrm{M}_{3}\left(L_{5}\right)$.

In Section 4 we will generalize these first two observations, and show how each yields an action of various symmetric groups as automorphisms of $\mathrm{M}_{d}\left(L_{n}\right)$, and hence of $L_{n}$, whenever $\operatorname{gcd}(d, n-1)=1$.

Third, and finally, it is somewhat less obvious that there are many other types of actions of various symmetric groups on $\mathrm{M}_{3}\left(L_{5}\right)$. To give one such example, here is yet another set of five matrices which, along with their duals, provides a set of generators for $\mathrm{M}_{3}\left(L_{5}\right)$. Loosely speaking, these are produced from the previous set $\left\{X_{1}, X_{2}, X_{3}, X_{4}, X_{5}\right\}$ by an appropriate 
permutation in $S_{5}$ together with an interchanging of the roles of the initial and final columns of $\mathrm{M}_{3}\left(L_{5}\right)$.

$$
\begin{gathered}
X_{1}=\left(\begin{array}{lll}
x_{1} x_{5} & 0 & 0 \\
x_{2} x_{5} & 0 & 0 \\
x_{3} x_{5} & 0 & 0
\end{array}\right) \quad X_{2}=\left(\begin{array}{ccc}
x_{4} & 0 & 0 \\
x_{4} x_{5} & 0 & 0 \\
x_{5}^{2} & 0 & 0
\end{array}\right) \\
X_{3}=\left(\begin{array}{lll}
x_{1} & 0 & 0 \\
x_{2} & 0 & 0 \\
x_{3} & 0 & 0
\end{array}\right) \quad X_{4}=\left(\begin{array}{ccc}
0 & 1 & 0 \\
0 & 0 & x_{2} \\
0 & 0 & x_{3}
\end{array}\right) \quad X_{5}=\left(\begin{array}{lll}
0 & 0 & x_{1} \\
0 & 0 & x_{4} \\
0 & 0 & x_{5}
\end{array}\right)
\end{gathered}
$$

We will describe subsequent to the proof of Theorem 4.14 a number of additional, significantly different collections of generating matrices in $\mathrm{M}_{d}\left(L_{n}\right)$ for $\operatorname{gcd}(d, n-1)=1$. Each of these collections gives rise to an automorphism of $\mathrm{M}_{d}\left(L_{n}\right)$. Because Theorem 4.14 will demonstrate that $\mathrm{M}_{d}\left(L_{n}\right) \cong L_{n}$ for $\operatorname{gcd}(d, n-1)=1$, each of these automorphisms of $\mathrm{M}_{d}\left(L_{n}\right)$ will in turn induce an automorphism of $L_{n}$.

\section{The Generators of $\mathrm{M}_{d}\left(L_{n}\right)$}

In this section we present the appropriate $2 n$ matrices of $\mathrm{M}_{d}\left(L_{n}\right)$ which generate $\mathrm{M}_{d}\left(L_{n}\right)$. We write $n=q d+r$ with $2 \leq r \leq d$. We assume $d<n$, so that $q \geq 1$. The matrices $X_{1}, X_{2}, \ldots, X_{q}$ are given as follows. For $1 \leq i \leq q$ we define

$$
X_{i}=\left(\begin{array}{cccc}
x_{(i-1) d+1} & 0 & & 0 \\
x_{(i-1) d+2} & 0 & & 0 \\
\vdots & 0 & \ldots & 0 \\
x_{i d} & 0 & & 0
\end{array}\right)=\sum_{j=1}^{d} x_{(i-1) d+j} e_{j, 1}
$$

The two matrices $X_{q+1}$ and $X_{q+2}$ play a pivotal role here. They are defined as follows.

$$
\begin{aligned}
X_{q+1}= & \left(\begin{array}{cccccccc}
x_{q d+1} & 0 & 0 & & 0 & 0 & & 0 \\
x_{q d+2} & 0 & 0 & & 0 & 0 & & 0 \\
\vdots & 0 & 0 & & 0 & 0 & & 0 \\
x_{n} & 0 & 0 & \ldots & 0 & 0 & \ldots & 0 \\
0 & 1 & 0 & & 0 & 0 & & 0 \\
0 & 0 & 1 & & 0 & 0 & & 0 \\
0 & 0 & 0 & \ldots & 1 & 0 & & 0
\end{array}\right) \\
& =\sum_{i=1}^{d-r} e_{i+r, i+1}+\sum_{t=1}^{r} x_{q d+t} e_{t, 1}
\end{aligned}
$$


and

$$
\begin{aligned}
& X_{q+2}=\left(\begin{array}{cccccccc}
0 & & 0 & 1 & 0 & 0 & 0 & 0 \\
0 & & 0 & 0 & 1 & 0 & 0 & 0 \\
& & & & & \vdots & & \\
0 & & 0 & 0 & 0 & 0 & 1 & 0 \\
0 & \ldots & 0 & 0 & 0 & 0 & 0 & a_{q+2, r-1} \\
0 & & 0 & 0 & 0 & 0 & 0 & a_{q+2, r} \\
& & & \vdots & & & & \vdots \\
0 & & 0 & 0 & 0 & 0 & 0 & a_{q+2, d}
\end{array}\right) \\
& =\sum_{j=1}^{r-2} e_{j, j+s}+\sum_{t=1}^{d-(r-2)} a_{q+2,(r-2)+t} e_{(r-2)+t, d}
\end{aligned}
$$

(where the elements $a_{q+2, r-1}, a_{q+2, r}, \ldots, a_{q+2, d} \in L_{n}$ are monomials in $x$-variables which will be determined later). In case $d-r=0$ or $r-2=0$ we interpret the appropriate sums as zero.

The remaining matrices $X_{q+3}, \ldots, X_{n}$ will be explicitly specified later, but each of these will have the same general form. In particular, for $q+3 \leq i \leq n$,

$$
X_{i}=\left(\begin{array}{cccc}
0 & & 0 & a_{i, 1} \\
0 & & 0 & a_{i, 2} \\
0 & \ldots & \vdots & \\
0 & & 0 & a_{i, d}
\end{array}\right)=\sum_{j=1}^{d} a_{i, j} e_{j, d}
$$

(where the elements $a_{i, 1}, a_{i, 2}, \ldots, a_{i, d} \in L_{n}$ are monomials in the $x$-variables which will be determined later). In case $q+3>n$ then we understand that there are no matrices of this latter form in our set of $2 n$ matrices. We note that we always have the matrices $X_{q+1}$ and $X_{q+2}$, since $n=q d+r \geq q \cdot 1+2$.

We define the matrices $Y_{i}$ for $1 \leq i \leq n$ by setting $Y_{i}=X_{i}^{*}$. Because they will play such an important role, we explicitly describe $Y_{q+1}$ and $Y_{q+2}$.

$$
\begin{aligned}
Y_{q+1}= & \left(\begin{array}{cccccccc}
y_{q d+1} & y_{q d+2} & \ldots & y_{n} & 0 & 0 & & 0 \\
0 & 0 & 0 & 0 & 1 & 0 & & 0 \\
0 & 0 & 0 & 0 & 0 & 1 & & 0 \\
& & & \vdots & & & \ldots & \\
0 & 0 & 0 & 0 & 0 & 0 & & 1 \\
0 & 0 & 0 & 0 & 0 & 0 & 0 \\
0 & 0 & 0 & 0 & 0 & 0 & & 0
\end{array}\right) \\
= & \sum_{i=1}^{d-r} e_{i+1, i+r}+\sum_{t=1}^{r} y_{q d+t} e_{1, t}
\end{aligned}
$$


and

$$
\begin{aligned}
& Y_{q+2}=\left(\begin{array}{cccccccc}
0 & 0 & & 0 & 0 & 0 & & 0 \\
& & & \vdots & & & \\
0 & 0 & & 0 & 0 & 0 & & 0 \\
1 & 0 & & 0 & 0 & 0 & \ldots & 0 \\
0 & 1 & & 0 & 0 & 0 & & 0 \\
& & \ldots & & & & & \vdots \\
0 & 0 & & 1 & 0 & 0 & & 0 \\
0 & 0 & & 0 & a_{q+2, r-1}^{*} & a_{q+2, r}^{*} & \ldots & a_{q+2, d}^{*}
\end{array}\right) \\
& =\sum_{j=1}^{r-2} e_{j+s, j}+\sum_{t=1}^{d-(r-2)} a_{q+2,(r-2)+t}^{*} e_{d,(r-2)+t}
\end{aligned}
$$

As above, in case $d-r=0$ or $r-2=0$ we interpret the corresponding sums as zero.

Definition 3.1. We denote by $A$ the subalgebra of $\mathrm{M}_{d}\left(L_{n}\right)$ generated by the matrices

$$
\left\{X_{i}, Y_{i} \mid 1 \leq i \leq n\right\}
$$

That is,

$$
A=<\left\{X_{i}, Y_{i} \mid 1 \leq i \leq n\right\}>
$$

So in order to achieve our main result, we seek to show that $A=\mathrm{M}_{d}\left(L_{n}\right)$.

Using the relation $\sum_{j=1}^{n} y_{j} x_{j}=1_{K}$, we immediately get

\section{Lemma 3.2.}

$$
\sum_{i=1}^{q+1} Y_{i} X_{i}=E_{s} \in A .
$$

A similar computation yields

\section{Lemma 3.3.}

(1) Assume the elements $\left\{a_{q+2, r-1}, \ldots, a_{q+2, d}\right\} \cup\left\{a_{i, j} \mid q+3 \leq i \leq n, 1 \leq j \leq d\right\}$ are chosen so that

$$
\sum_{i, j} a_{i, j}^{*} a_{i, j}=1_{K}
$$

Then

$$
\sum_{i=q+2}^{n} Y_{i} X_{i}=I-E_{s} \in A .
$$

(2) Assume the elements $\left\{a_{q+2, r-1}, \ldots, a_{q+2, d}\right\}$ are chosen so that

$$
a_{q+2, j} a_{q+2, i}^{*}=\delta_{i, j}
$$

for every $i, j \in\{r-1, \ldots, d\}$. Then $X_{q+2} Y_{q+2}=I$.

Lemma 3.4. If $a \in A$ then $a^{*} \in A$.

Proof. The set of generators of $A$ has this property, and the relations are self-dual, hence for any element $a$ which can be generated by ring-theoretic operations we can also generate $a^{*}$. 
Definition 3.5. Recall the partition $\hat{S}_{1} \cup \hat{S}_{2}$ of $\{1,2, \ldots, d\}$ described in Section 1. For $i, j \in\{1,2, \ldots, d\}$ we write $i \sim j$ in case $i, j$ are both in the same $\hat{S}_{k}, k=1,2$.

Our goal for the remainder of this section is to show that $A$ contains all matrix units $e_{i, j}$ for $i \sim j$. We begin by defining two monomorphisms of $\mathrm{M}_{d}\left(L_{n}\right)$ which will be useful in this context.

Definition 3.6. We define the monomorphism $\beta$ of $\mathrm{M}_{d}\left(L_{n}\right)$ by setting

$$
\beta(M)=Y_{q+1} M X_{q+1}
$$

for each $M \in \mathrm{M}_{d}\left(L_{n}\right)$. Since $Y_{q+1}$ and $X_{q+1}$ are each in $A$, then $\beta$ in fact restricts to a monomorphism of $A$.

Assuming that we have chosen the elements $\left\{a_{q+2, r-1}, \ldots, a_{q+2, d}\right\}$ as described in Lemma 3.3 (2), we define the monomorphism $\phi$ of $\mathrm{M}_{d}\left(L_{n}\right)$ by setting

$$
\phi(M)=Y_{q+2} M X_{q+2}
$$

for each $M \in \mathrm{M}_{d}\left(L_{n}\right)$. Since $Y_{q+2}$ and $X_{q+2}$ are each in $A$, then $\phi$ in fact restricts to a monomorphism of $A$.

We begin by showing that all of the matrix idempotents $\left\{e_{i} \mid 1 \leq i \leq d\right\}$ are in $A$. The results presented in the next two lemmas follow directly from straightforward matrix computations, so we omit their proofs.

Lemma 3.7. If $k<r-1$ then

$$
\phi\left(e_{k}\right)=e_{k+s}
$$

Lemma 3.8. If $k>r$ then

$$
\beta\left(e_{k}\right)=e_{k-(r-1)} \text {. }
$$

It is instructive to note the following. In words, the previous two lemmas say that we can move matrix idempotents "forward by $s$ " (if we start with an index less than $r-1$ ), and "backwards by $r-1$ " (if we start with an index bigger than $r$ ). But even though it would make sense to move the specific idempotent $e_{r-1}$ forward by $s$ units (since $(r-1)+s=d$ ), or to move the specific idempotent $e_{r}$ backwards by $r-1$ units, neither of these moves can be effected by the matrix multiplications described in the lemmas. For instance, the entry in the $(d, d)$ coordinate of $\phi\left(e_{r-1}\right)=Y_{q+2} e_{r-1} X_{q+2}$ is $a_{q+2, r-1}^{*} a_{q+2, r-1}$, which may or may not equal 1 depending on the choice of $a_{q+2, r-1}$. (Indeed, we will see later that we will NOT choose $a_{q+2, r-1}$ having this property.) This observation is precisely the reason why we must expend so much effort in analyzing the partition $\hat{S}_{1} \cup \hat{S}_{2}$ of $\{1,2, \ldots, d\}$ described previously.

We consider the sequence $\left\{u_{i}\right\}_{i=1}^{d}$ of integers, whose $i^{\text {th }}$ entry is given by

$$
u_{i}=i s(\bmod d) \text {. }
$$

The integers $u_{i}$ are understood to be taken from the set $\{1,2, \ldots, d\}$. Rephrased, we define the sequence $\left\{u_{i}\right\}_{i=1}^{d}$ by setting $u_{1}=s$, and, for $1 \leq i \leq d-1$,

$$
u_{i+1}=u_{i}+s \text { if } u_{i} \leq r-1, \quad \text { and } \quad u_{i+1}=u_{i}-(r-1) \text { if } u_{i} \geq r .
$$

Of course, the $u$-sequence is closely related to the $h$-sequence described in Section 1, Thus it is not surprising that the following Lemma closely resembles Lemma 1.5. Because $\operatorname{gcd}(d, s)=1$ (so that $s$ is invertible mod $d$ ), basic number theory yields the following 


\section{Lemma 3.9.}

(1) The entries in the sequence $u_{1}, u_{2}, \ldots, u_{d}$ are distinct.

(2) The set of entries $\left\{u_{1}, u_{2}, \ldots, u_{d}\right\}$ equals the set $\{1,2, \ldots, d\}$ (in some order).

(3) The penultimate entry in the sequence is $r-1$; that is, $u_{d-1}=r-1$.

(4) The final entry in the sequence is $d$; that is, $u_{d}=d$.

Proof. The only non-standard statements are (3) and (4). Suppose $r-1=i s(\bmod d)$. Then $d=r-1+s=(i+1) s(\bmod d)$. Now $\operatorname{gcd}(s, d)=1$ gives that $i+1=d$, so that $i=d-1$ and we get $r-1=(d-1) s(\bmod d)=u_{d-1}$ as desired. Then $(4)$ follows directly from $(3)$ and the equation $d=(r-1)+s$.

Proposition 3.10. For every $j$ with $1 \leq j \leq d$ we have $e_{j} \in A$.

Proof. The key idea is to show that $E_{j} \in A$ for all $1 \leq j \leq d$. Since $X_{1} Y_{1}=I$ we have $I=E_{d} \in A$. We consider the sequence of matrices $E_{u_{1}}, E_{u_{2}}, \ldots, E_{u_{d}}$ arising from the sequence $\left\{u_{i}\right\}_{i=1}^{d}$ described above. By induction on $i$, we show that each of $E_{u_{1}}, E_{u_{2}}, \ldots, E_{u_{d-1}} \in A$. For $i=1$ we have $E_{u_{1}}=E_{s} \in A$ by Lemma 3.2. Now we assume that $E_{u_{i}} \in A$ for $i \leq d-2$, and show that $E_{u_{i+1}} \in A$. By Lemma 3.9)(3), $i \leq d-2$ gives that $u_{i} \neq r-1$. There are two cases.

Case 1: $u_{i} \leq r-2$. Then by definition $u_{i+1}=u_{i}+s$. Since $E_{u_{i}} \in A$ by hypothesis, we have $\phi\left(E_{u_{i}}\right) \in A$, which then gives

$$
E_{s}+\phi\left(E_{u_{i}}\right) \in A
$$

But since $u_{i} \leq r-2$, Lemma 3.7 applies to give

$$
\phi\left(E_{u_{i}}\right)=\sum_{j=1}^{u_{i}} e_{j+s}=\sum_{k=s+1}^{u_{i}+s} e_{k}
$$

so that

$$
E_{s}+\phi\left(E_{u_{i}}\right)=\sum_{k=1}^{s} e_{k}+\sum_{k=s+1}^{u_{i}+s} e_{k}=E_{u_{i}+s}=E_{u_{i+1}},
$$

so that $E_{u_{i+1}} \in A$, and Case 1 is shown.

Case 2: $u_{i} \geq r$. Since $I=E_{d} \in A$ we have $I-E_{u_{i}} \in A$, and since $E_{s} \in A$ we get

$$
E_{s}-\beta\left(I-E_{u_{i}}\right) \in A \text {. }
$$

But $I-E_{u_{i}}=\sum_{j=u_{i}+1}^{d} e_{j}$, and $u_{i}+1>r$, so Lemma 3.8 applies to give

$$
\beta\left(I-E_{u_{i}}\right)=\sum_{j=u_{i}+1}^{d} e_{j-(r-1)}
$$


Thus we get that

$$
\begin{aligned}
E_{s}-\beta\left(I-E_{u_{i}}\right) & =E_{s}-\sum_{j=u_{i}+1}^{d} e_{j-(r-1)} \\
& =E_{s}-\sum_{k=u_{i}+1-(r-1)}^{d-(r-1)} e_{k}=E_{s}-\sum_{k=u_{i}+1-(r-1)}^{s} e_{k} \\
& =\sum_{k=1}^{u_{i}-(r-1)} e_{k}=E_{u_{i}-(r-1)}=E_{u_{i+1}},
\end{aligned}
$$

so that $E_{u_{i+1}} \in A$, and Case 2 is shown. Thus we have established by induction that $E_{u_{i}} \in A$ for all $1 \leq i \leq d-1$. But $E_{u_{d}}=E_{d}$ by Lemma [3.9, and $E_{d}=I \in A$ has already been established, so in fact we have $E_{u_{i}} \in A$ for all $1 \leq i \leq d$. So by Lemma 3.9)(2) we conclude that $E_{j} \in A$ for all $1 \leq j \leq d$.

Now the desired result follows easily from the observation that $e_{1}=E_{1} \in A$, while $e_{j}=$ $E_{j}-E_{j-1} \in A$ for all $2 \leq j \leq d$.

We remark that we need not modify the proof of Proposition 3.10 at all in case $r=2$ (resp. $r=d)$. This is because even though we would not have the matrix $X_{q+2}\left(\operatorname{resp} . X_{q+1}\right)$ containing 1 in the appropriate entries, in the case $r=2$ (resp. $r=d$ ) we would have $s=d-1$ (resp. $s=1$ ), so that we would only be using multiplication by $X_{q+1}\left(\right.$ resp. $\left.X_{q+2}\right)$ in the proof.

Now that we have established that all of the matrix idempotents $e_{i}(1 \leq i \leq d)$ are in $A$, we use them to generate all of the matrix units $e_{i, j}$.

\section{Lemma 3.11.}

(1) Suppose $1+s<d$. Then $e_{1} X_{q+2} e_{1+s}=e_{1,1+s}$, and $e_{1,1+s} \in A$.

(2) Suppose $1+s=d$. Then $\hat{S}_{1}=\{1\}$ and $\hat{S}_{2}=\{2, \ldots, d\}$.

(3) The situation $1+s>d$ is not possible.

Proof. (1) We have $1+s \leq d-1$. By construction, the $(1, s+1)$ entry of $X_{q+2}$ is 1 as long as $r-2 \geq 1$. But $1+s \leq d-1$ gives $d-(r-2) \leq d-1$, which yields the desired $r-2 \geq 1$. Now use Proposition 3.10 .

(2) If $1+s=d$, since $(r-1)+s=d$ we get $r-1=1$. So the sequence $\left\{h_{i}\right\}_{i=1}^{d}$ has $h_{1}=1=r-1$, so that $\hat{S}_{1}=\{1\}$.

(3) If $1+s>d$, then with $(r-1)+s=d$ we would get $r<2$, contradicting the hypothesis that $r \geq 2$.

\section{Lemma 3.12.}

(1) Suppose $n$ is not a multiple of $d$. Then $e_{d} X_{q+1} e_{s}=e_{d, s}$, and $e_{d, s} \in A$.

(2) Suppose $n$ is a multiple of $d$. Then $\hat{S}_{1}=\{1,2, \ldots, d-1\}$ and $\hat{S}_{2}=\{d\}$.

Proof. (1) If $n$ is not a multiple of $d$ then $r \neq d$, so that the $(d, s)$ entry of the matrix $X_{q+1}$ is 1. Now use Proposition 3.10.

(2) On the other hand, if $n$ is a multiple of $d$, then $n=q d+d$, so $r=d$, so that $r-1=d-1$, which gives $s=d-(r-1)=1$, so that the sequence $\left\{h_{i}\right\}_{i=1}^{d}$ has $h_{1}=1, h_{i}=h_{i}+1$, and the result follows. 
The next Proposition provides a link between the matrix units $e_{i, j} \in A$ and the partition $\hat{S}_{1} \cup \hat{S}_{2}$ of $\{1,2, \ldots, d\}$.

Proposition 3.13. Consider the sequence $\left\{h_{i}\right\}_{i=1}^{d}$ described in Section 1 . Let $h_{i}, h_{i+1}, h_{i+2}$ be three consecutive elements of the sequence, where $h_{i} \neq r, r-1$ and $h_{i+1} \neq r, r-1$. (In other words, consider three consecutive elements $h_{i}, h_{i+1}, h_{i+2}$ so that all three are in $\hat{S}_{1}$ or all three are in $\hat{S}_{2}$.) Then there exists $X \in\left\{X_{q+1}, X_{q+2}\right\}$ and $Y \in\left\{Y_{q+1}, Y_{q+2}\right\}$ so that

$$
Y e_{h_{i}, h_{i+1}} X=e_{h_{i+1}, h_{i+2}} .
$$

In particular, in this situation, if $e_{h_{i}, h_{i+1}} \in A$ then also $e_{h_{i+1}, h_{i+2}} \in A$.

Proof. There are four cases to consider, depending on whether we use the "plus $s$ " or "minus $r-1 "$ operation to get from one element of the sequence to the next.

Case 1: $h_{i+1}=h_{i}+s$ and $h_{i+2}=h_{i+1}+s$. In this situation we have $h_{i} \leq r-1$ because $h_{i+1} \leq d=s+(r-1)$ and $h_{i+1}=h_{i}+s$. But $h_{i} \neq r-1$ by hypothesis. Thus we have in fact $h_{i} \leq r-2$. In an exactly analogous way we also have $h_{i+1} \leq r-2$. Using that each of $h_{i}$ and $h_{i+1}$ is less than $r-1$, we get

$$
Y_{q+2} e_{h_{i}, h_{i+1}} X_{q+2}=e_{h_{i+1}, h_{i+2}} .
$$

Case 2: $h_{i+1}=h_{i}+s$ and $h_{i+2}=h_{i+1}-(r-1)$. As in Case 1 we have $h_{i}<r-1$. Also, $h_{i+1} \geq r$ because $1 \leq h_{i+2}=h_{i+1}-(r-1)$. But $h_{i+1} \neq r$ by hypothesis. Thus we have in fact $h_{i+1}>r$. Using both that $h_{i}<r-1$ and $h_{i+1}>r$, we get

$$
Y_{q+2} e_{h_{i}, h_{i+1}} X_{q+1}=e_{h_{i+1}, h_{i+2}} .
$$

Case 3: $h_{i+1}=h_{i}-(r-1)$ and $h_{i+2}=h_{i+1}+s$. As shown above, the hypotheses yield $h_{i}>r$ and $h_{i+1}<r-1$, from which we get

$$
Y_{q+1} e_{h_{i}, h_{i+1}} X_{q+2}=e_{h_{i+1}, h_{i+2}} .
$$

Case 4: $h_{i+1}=h_{i}-(r-1)$ and $h_{i+2}=h_{i+1}-(r-1)$. As shown above, the hypotheses yield $h_{i}>r$ and $h_{i+1}>r$, from which we get

$$
Y_{q+1} e_{h_{i}, h_{i+1}} X_{q+1}=e_{h_{i+1}, h_{i+2}}
$$

and the result is established.

We now establish the relationship between the partition $\hat{S}_{1} \cup \hat{S}_{2}$ of $\{1,2, \ldots, d\}$ and the matrix units $e_{i, j} \in A$. Intuitively, the idea is this. Suppose for instance that $a, b \in \hat{S}_{1}$. We seek to show that $e_{a, b} \in A$. There is a sequence of elements in $\hat{S}_{1}$ which starts at $a$ (resp. $b$ ) and ends at $r-1$. By the previous result, this will imply that $e_{a, r-1} \in A$ (resp. $e_{b, r-1} \in A$ ). But then by duality $e_{r-1, b} \in A$, so that $e_{a, r-1} e_{r-1, b}=e_{a, b} \in A$. Here are the formal details.

Proposition 3.14. Suppose $h_{i}, h_{j}$ are two entries in the sequence $\left\{h_{i}\right\}_{i=1}^{d}$, for which both entries are either in $\hat{S}_{1}$ or $\hat{S}_{2}$. Then

$$
e_{h_{i}, h_{j}} \in A
$$


Proof. We start by proving the result for $\hat{S}_{1}$. Suppose first that we are in a situation for which $1+s<d$. Then Lemma 3.11(1) yields that $e_{1,1+s} \in A$. Since in this situation the integers $1,1+s$ are the first two elements of the sequence $\left\{h_{i}\right\}_{i=1}^{d}$, and both are in $\hat{S}_{1}$, repeated applications of Proposition 3.13 gives that $e_{h_{i}, h_{i+1}} \in A$ for any two consecutive elements $h_{i}, h_{i+1}$ of $\hat{S}_{1}$. By matrix multiplication this then gives $e_{h_{i}, h_{j}} \in A$ whenever $i<j$ and both $h_{i}, h_{j}$ are in $\hat{S}_{1}$. By Lemma 3.4 this gives that $e_{h_{i}, h_{j}} \in A$ whenever $i \neq j$ and both $h_{i}, h_{j}$ are in $\hat{S}_{1}$. This together with Proposition 3.10 yields that $e_{h_{i}, h_{j}} \in A$ whenever both $h_{i}, h_{j}$ are in $\hat{S}_{1}$.

On the other hand, if we are in a situation for which $1+s=d$, then by Lemma 3.11(2) we have that $\hat{S}_{1}=\{1\}$, and the result follows immediately from Proposition 3.10 .

The result for $\hat{S}_{2}$ is established in a similar manner, using Lemma 3.12 and Propositions 3.10 and 3.13, along with the fact that whenever $n$ is not a multiple of $d$, then the first two elements of $\hat{S}_{2}$ in the sequence $\left\{h_{i}\right\}_{i=1}^{d}$ are $d, s$.

\section{THE MAIN THEOREM}

With the results of Section 3 in hand, we now show how the partition $\hat{S}_{1} \cup \hat{S}_{2}$ of $\{1,2, \ldots, d\}$ can be used to specify the elements of $X_{q+2}, \ldots, X_{n}$ in such a way that the set

$$
\left\{X_{1}, \ldots, X_{n}, Y_{1}, \ldots, Y_{n}\right\}
$$

generates $\mathrm{M}_{d}\left(L_{n}\right)$.

Definition 4.1. We define a partition $S_{1} \cup S_{2}$ of $\{1,2, \ldots, n\}$ as follows: For $w \in\{1,2, \ldots, n\}$, write $w=q_{w} d+\hat{w}$ with $1 \leq \hat{w} \leq d$. We then define $w \in S_{k}$ (for $k=1,2$ ) if and only if $\hat{w} \in \hat{S}_{k}$.

So we are 'enlarging' the partition of $\{1,2, \ldots, d\}=\hat{S}_{1} \cup \hat{S}_{2}$ to a partition of $\{1,2, \ldots, n\}=$ $S_{1} \cup S_{2}$ by extending modulo $d$.

Now consider this set, which we will call "The List":

$$
\begin{gathered}
x_{1}^{d-1} \\
x_{2} x_{1}^{d-2}, x_{3} x_{1}^{d-2}, \ldots, x_{n} x_{1}^{d-2} \\
x_{2} x_{1}^{d-3}, x_{3} x_{1}^{d-3}, \ldots, x_{n} x_{1}^{d-3} \\
\vdots \\
x_{2} x_{1}, x_{3} x_{1}, \ldots, x_{n} x_{1} \\
x_{2}, x_{3}, \ldots, x_{n}
\end{gathered}
$$

Lemma 4.2. The elements of The List satisfy $(\dagger)$. That is,

$$
y_{1}^{d-1} x_{1}^{d-1}+\sum_{i=0}^{d-2} \sum_{j=2}^{n} y_{1}^{i} y_{j} x_{j} x_{1}^{i}=1_{K} .
$$


Proof. We note that

$$
\begin{aligned}
y_{1}^{d-1} x_{1}^{d-1}+\sum_{i=0}^{d-2} \sum_{j=2}^{n} y_{1}^{i} y_{j} x_{j} x_{1}^{i} & =y_{1}^{d-1} x_{1}^{d-1}+\sum_{j=2}^{n} y_{1}^{d-2} y_{j} x_{j} x_{1}^{d-2}+\sum_{i=0}^{d-3} \sum_{j=2}^{n} y_{1}^{i} y_{j} x_{j} x_{1}^{i} \\
& =\sum_{j=1}^{n} y_{1}^{d-2} y_{j} x_{j} x_{1}^{d-2}+\sum_{i=0}^{d-3} \sum_{j=2}^{n} y_{1}^{i} y_{j} x_{j} x_{1}^{i} \\
& =y_{1}^{d-2}\left(\sum_{j=1}^{n} y_{j} x_{j}\right) x_{1}^{d-2}+\sum_{i=0}^{d-3} \sum_{j=2}^{n} y_{1}^{i} y_{j} x_{j} x_{1}^{i} \\
& =y_{1}^{d-2}\left(1_{K}\right) x_{1}^{d-2}+\sum_{i=0}^{d-3} \sum_{j=2}^{n} y_{1}^{i} y_{j} x_{j} x_{1}^{i} \\
& =y_{1}^{d-2} x_{1}^{d-2}+\sum_{i=0}^{d-3} \sum_{j=2}^{n} y_{1}^{i} y_{j} x_{j} x_{1}^{i} .
\end{aligned}
$$

By induction we continue in a similar way to get

$$
=y_{1} x_{1}+\sum_{j=2}^{n} y_{j} x_{j}=1_{K} .
$$

It is clear that

Lemma 4.3. There are $(d-1)(n-1)+1$ elements on The List.

Lemma 4.4. The number of entries $\left\{a_{q+2, r-1}, \ldots, a_{q+2, d}\right\} \cup\left\{a_{i, j} \mid q+3 \leq i \leq n, 1 \leq j \leq d\right\}$ which must be specified to form the matrices $X_{q+2}, X_{q+3}, \ldots, X_{n}$ is

$$
(s+1)+d[n-(q+2)] .
$$

Proof. The elements $\left\{a_{q+2, j} \mid r-1 \leq j \leq d\right\}$ needed to complete $X_{q+2}$ is a list containing $d-(r-1)+1=s+1$ entries. There are $n-(q+2)$ matrices in the list $X_{q+3}, \ldots, X_{n}$, and each of these matrices will contain exactly $d$ nonzero entries.

Lemma 4.5. The number of entries which must be specified to form the matrices $X_{q+2}, X_{q+3}, \ldots, X_{n}$ is equal to the number of entries in The List.

Proof. By Lemmas 4.3 and 4.4, we must show

$$
(s+1)+d[n-(q+2)]=(d-1)(n-1)+1 .
$$


But

$$
\begin{aligned}
(s+1)+d[n-(q+2)] & =[d-(r-1)]+1+d n-d q-2 d \\
& =d-r+2+d n-d q-2 d \\
& =-(n-q d)+2+d n-d q-d \\
& =n(d-1)+2-d \\
& =n(d-1)-(d-1)+1 \\
& =(n-1)(d-1)+1 .
\end{aligned}
$$

The following result describes exactly how many of the entries to be specified in $X_{q+2}, \ldots, X_{n}$ correspond to the subset $\hat{S}_{1}$ in the partition $\hat{S}_{1} \cup \hat{S}_{2}$ of $\{1,2, \ldots, d\}$.

Lemma 4.6. Consider the set of matrices $X_{q+3}, \ldots, X_{n}$, together with the last $s+1$ rows of $X_{q+2}$. Then the number of nonzero entries corresponding to rows indexed by elements of $\hat{S}_{1}$ equals

$$
d_{1}[n-(q+2)]+e_{1}
$$

Proof. This follows directly by an argument analogous to that given in the proof of Lemma 4.4, together with the definitions of $d_{1}$ and $e_{1}$.

Lemma 4.7. The number of entries on The List of the form $x_{u} x_{1}^{t}$ for which $u \in S_{1}$ is

$$
(d-1)\left[\left(q d_{1}-1\right)+f_{1}\right]+1 .
$$

Proof. Consider each of the $d-1$ rows of The List (other than the first). For each of the $d_{1}$ entries which are in $\hat{S}_{1}$ (including 1 ) there are $q$ elements congruent to it (modulo $d$ ). So we get $q d_{1}$ such entries. But we have started each list with $x_{2}$ (and not $x_{1}$ ), so in fact there are $q d_{1}-1$ such entries in each row. Each row also contains $f_{1}$ entries from the set $\{q d+1, \ldots, q d+r=n\}$. There are $d-1$ rows. Finally, we add in the term corresponding to $x_{1}^{d-1}$.

Before we get to the main proposition, we need a computational lemma.

\section{Lemma 4.8.}

$$
d_{1} r=d f_{1}-d+d_{1}+1
$$

Proof. Using the equations $d_{1}=1+b+t$ and $(1+t)(r-1)=1+b s$ from Proposition 1.7 , we get

$$
\begin{aligned}
d_{1} r & =(1+b+t) r=(1+t) r+b r \\
& =(1+t)(r-1)+(1+t)+b r \\
& =b s+b r+t+2
\end{aligned}
$$

while

$$
\begin{aligned}
d f_{1}-d+d_{1}+1 & =(s+(r-1))(1+b)-(s+(r-1))+(1+b+t)+1 \\
& =s b+r b+t+2 \quad \text { (by an easy computation). }
\end{aligned}
$$


We are now ready to prove the key algorithmic tool which will provide the vehicle for our main result.

Proposition 4.9. Consider the set of matrices $X_{q+3}, \ldots, X_{n}$, together with the last $s+1$ rows of $X_{q+2}$. Then the number of nonzero entries corresponding to rows indexed by elements of $\hat{S}_{1}$ equals the number of entries on The List of the form $x_{u} x_{1}^{t}$ for which $u \in S_{1}$.

Rephrased: It is possible to place the elements of The List in the "to be specified" entries of the matrices $X_{q+2}, X_{q+3}, \ldots, X_{n}$ in such a way that each entry of the form $x_{u} x_{1}^{t}$ for $u \in S_{k}$ $(k=1,2)$ is placed in a row indexed by $\hat{u}$ where $\hat{u} \in \hat{S}_{k}(k=1,2)$.

Proof. By Lemmas 4.6 and 4.7 it suffices to show that

$$
d_{1}[n-(q+2)]+e_{1}=(d-1)\left[\left(q d_{1}-1\right)+f_{1}\right]+1 .
$$

But

$$
\begin{aligned}
d_{1}[n-(q+2)]+e_{1} & =d_{1}[q d+r-q-2]+e_{1} \\
& =d_{1} q(d-1)+d_{1} r-2 d_{1}+e_{1} \\
& =d_{1} q(d-1)+\left[d f_{1}-d+d_{1}+1\right]-2 d_{1}+e_{1} \text { (using Lemma 4.8) } \\
& =d_{1} q(d-1)+\left[d f_{1}-d+d_{1}+1\right]-2 d_{1}+\left[d_{1}+1-f_{1}\right] \text { (Definition 1.6) } \\
& =d_{1} q(d-1)+(d-1) f_{1}-(d-1)+1 \\
& =(d-1)\left[d_{1} q-1+f_{1}\right]+1
\end{aligned}
$$

and we are done.

In other words, Proposition 4.9 implies that is possible to place the entries of The List in the empty "boxes" of the matrices $X_{q+2}, X_{q+3}, \ldots, X_{n}$ in such a way that each entry of the form $x_{u} x_{1}^{t}$ for $u \in S_{k}(k=1,2)$ is placed in a row indexed by $\hat{u}$ where $\hat{u} \in \hat{S}_{k}(k=1,2)$.

We assume for the remainder of this article that we have made such a placement. To help the reader clarify the process, a specific example appears below. However, the reader should keep in mind that in fact there are many possible such placements.

Once such a placement has been made, we can immediately deduce various properties of the matrices $\left\{X_{1}, \ldots, X_{n}, Y_{1}, \ldots, Y_{n}\right\}$. For instance,

Lemma 4.10. For all $1 \leq i, j \leq n$ we have

$$
X_{i} Y_{j}=\delta_{i, j} I \text { in } \mathrm{M}_{d}\left(L_{n}\right) .
$$

Proof. By definition of the matrices $X_{i}, Y_{j}$ it suffices to show that

$$
x_{i} x_{1}^{t} \cdot y_{1}^{u} y_{j}=\delta_{t, u} \delta_{i, j} 1_{K}
$$

for all $1 \leq i, j \leq n$ and $1 \leq u, t \leq d-2$. But this follows easily by the definition of multiplication in $L_{n}$.

Lemma 4.11. For each $w$ having $1 \leq w \leq n, x_{w} e_{\hat{w}, 1} \in A$ where $w \sim \hat{w}$.

Proof. Write $w=q_{w} d+\hat{w}$ with $1 \leq \hat{w} \leq d$. But $e_{\hat{w}}$ and $e_{1}$ are in $A$, so $e_{\hat{w}} X_{q_{w}} e_{1} \in A$, and this gives the result.

Lemma 4.12. For each $v$ having $1 \leq v \leq n, y_{v} e_{1, \hat{v}} \in A$ where $v \sim \hat{v}$. 
Proof. Write $v=q_{v} d+\hat{v}$ with $1 \leq \hat{v} \leq d$. But $e_{\hat{v}}$ and $e_{1}$ are in $A$, so $e_{1} Y_{q_{v}} e_{\hat{v}} \in A$, and this gives the result.

(We note that indeed Lemma 4.12 can also be established directly from Lemmas 4.11 and 3.4.)

Proposition 3.14 yields that matrix units indexed by the sets $\hat{S}_{1}$ and $\hat{S}_{2}$ are in $A$. In order to show that all the matrix units $\left\{e_{i, j} \mid 1 \leq i, j \leq d\right\}$ are in $A$, we need to provide a "bridge" between these two subsets of matrix units. That connection is made in the following Proposition, which provides the last major piece of the puzzle.

\section{Proposition 4.13.}

$$
e_{1, d} \in A \text { and } e_{d, 1} \in A \text {. }
$$

Proof. Because we have assumed that we have placed the elements from The List in a manner ensured by Proposition 4.9, there exists $M \in\left\{X_{q+2}, X_{q+3}, \ldots, X_{n}\right\}$ and an integer $l \in\{1,2, \ldots, d\}$ for which $l \sim 1$, and for which the $(l, d)$ entry of $M$ is $x_{1}^{d-1}$. That is, $e_{l} M e_{d}=x_{1}^{d-1} e_{l, d} \in A$. But because $l \sim 1$, Proposition 3.14 gives that $e_{1, l} \in A$. Thus $e_{1, l} x_{1}^{d-1} e_{l, d} \in A$, so

$$
x_{1}^{d-1} e_{1, d} \in A .
$$

We have $y_{1} e_{1}=e_{1} Y_{1} e_{1} \in A$, so that

$$
y_{1} e_{1} \cdot x_{1}^{d-1} e_{1, d}=y_{1} x_{1}^{d-1} e_{1, d}=y_{1} x_{1} x_{1}^{d-2} e_{1, d} \in A .
$$

Now choose any $w$ with $2 \leq w \leq n$. Again using the hypothesis that we have placed the elements from The List in a manner ensured by Proposition 4.9, there exists $M \in$ $\left\{X_{q+2}, X_{q+3}, \ldots, X_{n}\right\}$ and $w^{\prime} \in\{1, \ldots, d\}$ for which $w^{\prime} \sim w$, and

$$
e_{w^{\prime}} M e_{d}=x_{w} x_{1}^{d-2} e_{w^{\prime}, d} \in A .
$$

Write $w=q_{w} d+\hat{w}$ with $1 \leq \hat{w} \leq d$. Then $w \sim \hat{w}$ by definition, and so we get $w^{\prime} \sim \hat{w}$. So by Proposition 3.14, $e_{\hat{w}, w^{\prime}} \in A$. In addition, $e_{1} Y_{q_{w}} e_{\hat{w}}=y_{w} e_{1, \hat{w}} \in A$. So we get

$$
y_{w} e_{1, \hat{w}} e_{\hat{w}, w^{\prime}} x_{w} x_{1}^{d-2} e_{w^{\prime}, d} \in A,
$$

so that $y_{w} x_{w} x_{1}^{d-2} e_{1, d} \in A$ for each $w$ having $2 \leq w \leq d$. This, together with the previously established $y_{1} x_{1} x_{1}^{d-2} e_{1, d} \in A$, gives

$$
\sum_{w=1}^{n} y_{w} x_{w} x_{1}^{d-2} e_{1, d}=\left(\sum_{w=1}^{n} y_{w} x_{w}\right) x_{1}^{d-2} e_{1, d}=1_{K} \cdot x_{1}^{d-2} e_{1, d} \in A
$$

so that

$$
x_{1}^{d-2} e_{1, d} \in A .
$$

By a procedure analogous to the one we have just completed, which shows how to obtain $x_{1}^{d-2} e_{1, d} \in A$ starting from $x_{1}^{d-1} e_{1, d} \in A$, we can show that each of the elements

$$
x_{1}^{d-3} e_{1, d}, x_{1}^{d-4} e_{1, d}, \ldots, x_{1} e_{1, d} \in A,
$$

the last of which similarly gives $\left(\sum_{w=1}^{n} y_{w} x_{w}\right) e_{1, d} \in A$, which then finally yields

$$
e_{1, d} \in A
$$

as desired. That $e_{d, 1} \in A$ follows from Lemma 3.4. 
We finally are in a position to prove the main result of this article.

Theorem 4.14. Let $d, n$ be positive integers, and $K$ any field. Let $L_{K, n}=L_{n}$ denote the Leavitt algebra of type $(1, n-1)$ with coefficients in $K$. Then $L_{n} \cong \mathrm{M}_{d}\left(L_{n}\right)$ if and only if $\operatorname{gcd}(d, n-1)=1$.

Proof. By Proposition 2.1, if $\operatorname{gcd}(d, n-1)>1$ then the module type of $\mathrm{M}_{d}\left(L_{n}\right)$ is not $(1, n-1)$, so that $\mathrm{M}_{d}\left(L_{n}\right)$ and $L_{n}$ cannot be isomorphic in this case.

For the implication of interest, suppose $\operatorname{gcd}(d, n-1)=1$, and suppose $d<n$. By Corollary 1.2, we need only show that the set $A=\left\{X_{1}, \ldots, X_{n}, Y_{1}, \ldots, Y_{n}\right\}$ satisfies the three indicated properties. That $X_{i} Y_{j}=\delta_{i, j} I$ follows directly by the definition of these matrices and Lemma 4.10. The equation $\sum_{j=1}^{n} Y_{j} X_{j}=I$ follows from Lemmas 3.2, 3.3, and 4.2.

For the final property, we must show that $A=<\left\{X_{1}, \ldots, X_{n}, Y_{1}, \ldots, Y_{n}\right\}>=\mathrm{M}_{d}\left(L_{n}\right)$. It suffices to show that $x_{w} e_{i, j} \in A$ for all $1 \leq w \leq n$ and all $i, j \in\{1,2, \ldots, d\}$, since by Lemma 3.4 this will yield $y_{w} e_{i, j} \in A$ for all $1 \leq w \leq n$ and all $i, j \in\{1,2, \ldots, d\}$, and these two collections together clearly generate all of $\mathrm{M}_{d}\left(L_{n}\right)$

By Proposition 4.9 we may assume that the elements from The List have been placed appropriately in the matrices $X_{q+2}, \ldots, X_{n}$. Now let $i, j \in\{1,2, \ldots, d\}$. If $i \sim j$ then $e_{i, j} \in A$ by Proposition 3.14. So suppose $i \in \hat{S}_{1}$ and $j \in \hat{S}_{2}$. Then $i \sim 1$ and $j \sim d$, so $e_{i, 1}$ and $e_{d, j}$ are each in $A$, again by Proposition 3.14. But Proposition 4.13 yields $e_{1, d} \in A$, so that

$$
e_{i, 1} e_{1, d} e_{d, j}=e_{i, j} \in A \text {. }
$$

The situation where $i \in \hat{S}_{2}$ and $j \in \hat{S}_{1}$ is identical, and thus yields $e_{i, j} \in A$ for all $i, j \in$ $\{1,2, \ldots, d\}$. Finally, since each of the elements $\left\{x_{w} \mid 1 \leq w \leq n\right\}$ is contained as an entry in one of the matrices $X_{1}, \ldots, X_{q+1}$, we can indeed generate all elements of the desired form in $A$. Thus we have shown that for $\operatorname{gcd}(d, n-1)=1$ and $d<n$ we have $L_{n} \cong \mathrm{M}_{d}\left(L_{n}\right)$.

To finish the proof of our main result we need only show that the desired isomorphism holds in case $d \geq n$. Write $d=q^{\prime}(n-1)+d^{\prime}$ with $1 \leq d^{\prime} \leq n-1$. Then easily $\operatorname{gcd}\left(d^{\prime}, n-1\right)=1$, so the previous paragraph yields $L_{n} \cong \mathrm{M}_{d^{\prime}}\left(L_{n}\right)$. But then also $d \equiv d^{\prime}(\bmod n-1)$, so by Proposition 1.1 (1) we get $\mathrm{M}_{d}\left(L_{n}\right) \cong \mathrm{M}_{d^{\prime}}\left(L_{n}\right) \cong L_{n}$, and we are done.

Notice that Theorem 4.14 does not depend on the choice of the positions of the elements from The List in the non-specified entries of the matrices $X_{q+2}, \ldots, X_{n}$, other than that the positions are consistent with the condition allowed by Proposition 4.9.

Example 4.15. We indicated in Section 2 that $L_{5} \cong \mathrm{M}_{3}\left(L_{5}\right)$; in fact, we provided there five different sets of appropriate generating matrices of $\mathrm{M}_{3}\left(L_{5}\right)$. Here is yet another set, built by using the recipe provided in Theorem 4.14. In this case we have $n=5, d=3, r=2, r-1=$ $1, s=3-1=2, \hat{S}_{1}=\{1\}, \hat{S}_{2}=\{2,3\}, S_{1}=\{1,4\}, S_{2}=\{2,3,5\}$. The List consists of the $(n-1)(d-1)+1=9$ elements $\left\{x_{1}^{2}, x_{2} x_{1}, x_{3} x_{1}, x_{4} x_{1}, x_{5} x_{1}, x_{2}, x_{3}, x_{4}, x_{5}\right\}$. The point to be made here is that only the elements $x_{1}^{2}, x_{4} x_{1}$, and $x_{4}$ can be placed in row 1 of column 3 , since $S_{1}=\{1,4\}$.

$$
X_{1}=\left(\begin{array}{lll}
x_{1} & 0 & 0 \\
x_{2} & 0 & 0 \\
x_{3} & 0 & 0
\end{array}\right) \quad X_{2}=\left(\begin{array}{ccc}
x_{4} & 0 & 0 \\
x_{5} & 0 & 0 \\
0 & 1 & 0
\end{array}\right)
$$




$$
X_{3}=\left(\begin{array}{ccc}
0 & 0 & x_{4} \\
0 & 0 & x_{3} x_{1} \\
0 & 0 & x_{2}
\end{array}\right) \quad X_{4}=\left(\begin{array}{ccc}
0 & 0 & x_{1}^{2} \\
0 & 0 & x_{2} x_{1} \\
0 & 0 & x_{3}
\end{array}\right) \quad X_{5}=\left(\begin{array}{ccc}
0 & 0 & x_{4} x_{1} \\
0 & 0 & x_{5} \\
0 & 0 & x_{5} x_{1}
\end{array}\right)
$$

We finish this section by describing some automorphisms of $L_{n}$ which arise as a consequence of Theorem 4.14. There are many possible assignments of the elements on The List to the "boxes" of the matrices $X_{q+2}, X_{q+3}, \ldots, X_{n}$ consistent with the method described in Proposition 4.9. In particular, this freedom of assignment affords an action of the bisymmetric group $S_{d_{1}} \times S_{d_{2}}$ on each of the matrices $X_{q+3}, \ldots, X_{n}$ by permuting the entries inside $\hat{S}_{1}$ and $\hat{S}_{2}$. Similarly, we have an action of $S_{e_{1}} \times S_{e_{2}}$ on $X_{q+2}$. This freedom of assignment also allows an action on each of the $d$ rows in the generating matrices. Specifically, for each row $i(1 \leq i \leq d)$, we can permute the $(i, d)$-entries of the $n-(q+2)$ matrices $X_{q+3}, \ldots, X_{n}$; each of the $d \cdot(n-(q+2))$ ! such permutations will yield a different set of generators for $\mathrm{M}_{d}\left(L_{n}\right)$. Thus we have described

$$
d \cdot(n-(q+2)) ! e_{1} ! e_{2} !\left(d_{1} ! d_{2} !\right)^{n-(q+2)}
$$

permutations on the entries of the matrices $X_{q+2}, X_{q+3}, \ldots, X_{n}$, each of which induces a distinct automorphism of $\mathrm{M}_{d}\left(L_{n}\right)$. In turn, by Theorem 4.14, each then induces an automorphism of $L_{n}$ whenever $\operatorname{gcd}(d, n-1)=1$. These permutations yield automorphisms on $L_{n}$ which generalize the specific automorphisms of $\mathrm{M}_{3}\left(L_{5}\right)$ described in Section 2 .

Intriguingly, the types of automorphisms described here and in Section 2 still do not in general completely describe all the automorphisms of $L_{n}$ which arise from producing appropriate sets of generators in $\mathrm{M}_{d}\left(L_{n}\right)$. We present here two additional specific examples of generating sets inside various-sized matrix rings. In both cases, the entries used to build the generating matrices $X_{1}, \ldots, X_{n}, Y_{1}, \ldots, Y_{n}$ are monomials of degree at most 2 . In contrast to the previously presented examples, because The List contains monomials of degree up to and including $d-1$, the examples given here cannot be realized as arising from automorphisms induced by permutations of the entries of a specific set of generators as constructed in Theorem 4.14.

Example: A set of generators of $\mathrm{M}_{4}\left(L_{6}\right) \cong L_{6}$. (So $d=4, d-1=3$; note that there are no monomials of degree 3 used in this set.)

$$
\begin{aligned}
X_{1}=\left(\begin{array}{llll}
x_{1} & 0 & 0 & 0 \\
x_{2} & 0 & 0 & 0 \\
x_{3} & 0 & 0 & 0 \\
x_{4} & 0 & 0 & 0
\end{array}\right) & X_{2}=\left(\begin{array}{cccc}
x_{5} & 0 & 0 & 0 \\
x_{6} & 0 & 0 & 0 \\
0 & 1 & 0 & 0 \\
0 & 0 & 1 & 0
\end{array}\right) \quad X_{3}=\left(\begin{array}{cccc}
0 & 0 & 0 & x_{1}^{2} \\
0 & 0 & 0 & x_{2} x_{1} \\
0 & 0 & 0 & x_{3} x_{1} \\
0 & 0 & 0 & x_{4} x_{1}
\end{array}\right) \\
X_{4}=\left(\begin{array}{llll}
0 & 0 & 0 & x_{5} x_{1} \\
0 & 0 & 0 & x_{6} x_{1} \\
0 & 0 & 0 & x_{1} x_{2} \\
0 & 0 & 0 & x_{2}^{2}
\end{array}\right) & X_{5}=\left(\begin{array}{llll}
0 & 0 & 0 & x_{3} x_{2} \\
0 & 0 & 0 & x_{4} x_{2} \\
0 & 0 & 0 & x_{5} x_{2} \\
0 & 0 & 0 & x_{6} x_{2}
\end{array}\right)
\end{aligned}
$$

Example: A set of generators of $\mathrm{M}_{5}\left(L_{9}\right) \cong L_{9}$. (So $d=5, d-1=4$; note that there are no monomials of degree 3 or 4 used in this set. Also note that, unlike the matrices constructed 
in Theorem 4.14, there are entries other than $1_{K}$ in column 2.)

$$
\begin{aligned}
& X_{1}=\left(\begin{array}{ccccc}
x_{1} & 0 & 0 & 0 & 0 \\
x_{2} & 0 & 0 & 0 & 0 \\
x_{3} & 0 & 0 & 0 & 0 \\
x_{4} & 0 & 0 & 0 & 0 \\
x_{5} & 0 & 0 & 0 & 0
\end{array}\right) \quad X_{2}=\left(\begin{array}{ccccc}
x_{6} & 0 & 0 & 0 & 0 \\
x_{7} & 0 & 0 & 0 & 0 \\
x_{8} & 0 & 0 & 0 & 0 \\
x_{9} & 0 & 0 & 0 & 0 \\
0 & x_{9} & 0 & 0 & 0
\end{array}\right) \quad X_{3}=\left(\begin{array}{ccccc}
0 & x_{1} & 0 & 0 & 0 \\
0 & x_{2} & 0 & 0 & 0 \\
0 & x_{3} & 0 & 0 & 0 \\
0 & x_{4} & 0 & 0 & 0 \\
0 & x_{5} & 0 & 0 & 0
\end{array}\right) \\
& X_{4}=\left(\begin{array}{ccccc}
0 & x_{6} & 0 & 0 & 0 \\
0 & x_{7} & 0 & 0 & 0 \\
0 & x_{8} & 0 & 0 & 0 \\
0 & 0 & 1 & 0 & 0 \\
0 & 0 & 0 & 1 & 0
\end{array}\right) \quad X_{5}=\left(\begin{array}{ccccc}
0 & 0 & 0 & 0 & x_{1}^{2} \\
0 & 0 & 0 & 0 & x_{2} x_{1} \\
0 & 0 & 0 & 0 & x_{3} x_{1} \\
0 & 0 & 0 & 0 & x_{4} x_{1} \\
0 & 0 & 0 & 0 & x_{5} x_{1}
\end{array}\right) \quad X_{6}=\left(\begin{array}{ccccc}
0 & 0 & 0 & 0 & x_{6} x_{1} \\
0 & 0 & 0 & 0 & x_{7} x_{1} \\
0 & 0 & 0 & 0 & x_{8} x_{1} \\
0 & 0 & 0 & 0 & x_{9} x_{1} \\
0 & 0 & 0 & 0 & x_{9}
\end{array}\right) \\
& X_{7}=\left(\begin{array}{ccccc}
0 & 0 & 0 & 0 & x_{1} x_{2} \\
0 & 0 & 0 & 0 & x_{2}^{2} \\
0 & 0 & 0 & 0 & x_{3} x_{2} \\
0 & 0 & 0 & 0 & x_{4} x_{2} \\
0 & 0 & 0 & 0 & x_{5} x_{2}
\end{array}\right) \quad X_{8}=\left(\begin{array}{ccccc}
0 & 0 & 0 & 0 & x_{6} x_{2} \\
0 & 0 & 0 & 0 & x_{7} x_{2} \\
0 & 0 & 0 & 0 & x_{8} x_{2} \\
0 & 0 & 0 & 0 & x_{8} \\
0 & 0 & 0 & 0 & x_{9} x_{2}
\end{array}\right) \quad X_{9}=\left(\begin{array}{ccccc}
0 & 0 & 0 & 0 & x_{6} \\
0 & 0 & 0 & 0 & x_{7} \\
0 & 0 & 0 & 0 & x_{3} \\
0 & 0 & 0 & 0 & x_{4} \\
0 & 0 & 0 & 0 & x_{5}
\end{array}\right)
\end{aligned}
$$

\section{Applichtions to $\mathrm{C}^{*}$-Algebras and questions about $K_{0}$}

As mentioned in the Introduction, one consequence of our main result is that we are able to directly and explicitly establish an affirmative answer to the question posed in [14], page 8, regarding isomorphisms between matrix rings over Cuntz algebras.

Theorem 5.1. $\mathrm{M}_{d}\left(\mathcal{O}_{n}\right) \cong \mathcal{O}_{n}$ if and only if $\operatorname{gcd}(d, n-1)=1$.

Proof. If $\operatorname{gcd}(d, n-1) \neq 1$, Then $M_{d}\left(\mathcal{O}_{n}\right) \nRightarrow \mathcal{O}_{n}$ by [14], Corollary 2.4.

So suppose conversely that $\operatorname{gcd}(d, n-1)=1$. Let $\left\{s_{1}, \ldots, s_{n}\right\} \subset \mathcal{O}_{n}$ be the orthogonal isometries generating $\mathcal{O}_{n}$. These satisfy:

(i) For every $1 \leq i, j \leq n, s_{i}^{*} s_{j}=\delta_{i, j}$, and

(ii) $1=\sum_{i=1}^{n} s_{i} s_{i}^{*}$.

Now consider the complex Leavitt algebra $L_{\mathbb{C}, n}$, and notice that by Proposition $1.1(2)$ there exists a (unique) $\mathbb{C}$-algebra morphism

$$
\varphi: L_{\mathbb{C}, n} \rightarrow \mathcal{O}_{n}
$$

given by the extension of the assignment $x_{i} \mapsto s_{i}^{*}$ and $y_{i} \mapsto s_{i}$ for $1 \leq i \leq n$. Since $L_{\mathbb{C}, n}$ is a simple algebra, $L_{\mathbb{C}, n} \cong \varphi\left(L_{\mathbb{C}, n}\right)$. But $\mathcal{P}_{n}=\varphi\left(L_{\mathbb{C}, n}\right)$ is the complex dense $*$-subalgebra of $\mathcal{O}_{n}$ generated by $\left\{s_{1}, \ldots, s_{n}\right\}$ (as a complex algebra). Now consider the morphism

$$
\varphi_{d}: M_{d}\left(L_{\mathbb{C}, n}\right) \rightarrow M_{d}\left(\mathcal{O}_{n}\right)
$$

induced by $\varphi$. (In particular, $\varphi_{d}\left(e_{i, j}\right)=e_{i, j}$ for each matrix unit $e_{i, j}, 1 \leq i, j \leq d$.) Notice that, if $X_{i}(1 \leq i \leq n)$ is any of the matrices defined in Section 3 then, by definition of the elements of The List, $\varphi_{d}\left(Y_{i}\right)=\varphi_{d}\left(X_{i}\right)^{*}$ with respect to the involution $*$ of $M_{d}\left(\mathcal{O}_{n}\right)$. So, by 
defining $S_{i}=\varphi_{d}\left(Y_{i}\right)$, we get $S_{i}^{*}=\varphi_{d}\left(X_{i}\right)$, and thus $\left\{S_{1}, \ldots, S_{n}\right\} \subset M_{d}\left(\mathcal{O}_{n}\right)$ is a family of $n$ orthogonal isometries satisfying $I_{d}=\sum_{i=1}^{n} S_{i} S_{i}^{*}$. Hence, by [9], Theorem 1.12, there exists an isomorphism

$$
\Phi: \mathcal{O}_{n} \rightarrow C^{*}\left(S_{1}, \ldots, S_{n}\right) \subseteq M_{d}\left(\mathcal{O}_{n}\right)
$$

defined by the rule $\Phi\left(s_{i}\right)=S_{i}$ for every $1 \leq i \leq n$. Now, applying Theorem 4.14 to $\mathcal{P}_{n}$ and $M_{d}\left(\mathcal{P}_{n}\right)$ (via $\varphi$ ), for every $1 \leq i, j \leq d$ and for every $1 \leq k \leq n$ we have

$$
s_{k} e_{i, j}=\varphi_{d}\left(y_{k} e_{i, j}\right) \in C^{*}\left(S_{1}, \ldots, S_{n}\right)
$$

so that the generators of $M_{d}\left(\mathcal{O}_{n}\right)$ lie in $C^{*}\left(S_{1}, \ldots, S_{n}\right)$. Thus, $\mathcal{O}_{n} \cong M_{d}\left(\mathcal{O}_{n}\right)$ via $\Phi$, so we are done.

As mentioned previously, the affirmative answer to the isomorphism question for matrix rings over Cuntz algebras provided in Theorem 5.1 is indeed already known, a byproduct of [15], Theorem 4.3(1). However, the method we have provided in Theorem 5.1 is significantly more elementary, and provides an explicit description of the germane isomorphisms (such an explicit description has previously not been known).

A second interesting consequence of Theorem 4.14 is that the class of matrices over Leavitt algebras is classifiable using K-theoretic invariants. (For additional information about purely infinite simple algebras and their $K$-theory, see [6].)

Theorem 5.2. Let $\mathcal{L}$ denote the set of purely infinite simple $K$-algebras

$$
\left\{\mathrm{M}_{d}\left(L_{n}\right) \mid d, n \in \mathbb{N}\right\} .
$$

Let $B, B^{\prime} \in \mathcal{L}$. Then $B \cong B^{\prime}$ if and only if there is an isomorphism $\phi: K_{0}(B) \rightarrow K_{0}\left(B^{\prime}\right)$ for which $\phi\left(\left[1_{B}\right]\right)=\left[1_{B^{\prime}}\right]$.

Proof. It is well known (see e.g. [19], page 5) that any unital isomorphism $f: B \rightarrow B^{\prime}$ induces a group isomorphism $K_{0}(f): K_{0}(B) \rightarrow K_{0}\left(B^{\prime}\right)$ sending $\left[1_{B}\right]$ to $\left[1_{B^{\prime}}\right]$.

To see the converse, first notice that, for any $B \in \mathcal{L}, B=\mathrm{M}_{d}\left(L_{n}\right)$ for suitable $d, n \in \mathbb{N}$. It is well known that

$$
\left(K_{0}\left(\mathrm{M}_{d}\left(L_{n}\right)\right),\left[1_{\mathrm{M}_{d}\left(L_{n}\right)}\right]\right) \cong(\mathbb{Z} /(n-1) \mathbb{Z},[d])
$$

(see e.g [7] or [6]). Hence, if $B^{\prime}=\mathrm{M}_{k}\left(L_{m}\right)$ for suitable $k, m \in \mathbb{N}$, then the existence of an isomorphism $\phi: K_{0}(B) \rightarrow K_{0}\left(B^{\prime}\right)$ forces that $n=m$.

Now, since every automorphism of $\mathbb{Z} /(n-1) \mathbb{Z}$ is given by multiplication by an element $1 \leq l \leq n-1$ such that $\operatorname{gcd}(l, n-1)=1$, the hypothesis $\phi\left(\left[1_{B}\right]\right)=\left[1_{B^{\prime}}\right]$ yields that $[k]=[d l] \in \mathbb{Z} /(n-1) \mathbb{Z}$, i.e., that $k \equiv d l(\bmod n-1)$. So Proposition $1.1(1)$ gives that

$$
\mathrm{M}_{k}\left(L_{n}\right) \cong \mathrm{M}_{d l}\left(L_{n}\right) \cong \mathrm{M}_{d}\left(\mathrm{M}_{l}\left(L_{n}\right)\right) .
$$

Since $\operatorname{gcd}(l, n-1)=1$, we have $\mathrm{M}_{l}\left(L_{n}\right) \cong L_{n}$ by Theorem 4.14. Hence, $\mathrm{M}_{d}\left(\mathrm{M}_{l}\left(L_{n}\right)\right) \cong$ $\mathrm{M}_{d}\left(L_{n}\right)$, whence

$$
\mathrm{M}_{k}\left(L_{n}\right) \cong \mathrm{M}_{d l}\left(L_{n}\right) \cong \mathrm{M}_{d}\left(\mathrm{M}_{l}\left(L_{n}\right)\right) \cong \mathrm{M}_{d}\left(L_{n}\right)
$$

as desired.

A significantly more general $\mathrm{C}^{*}$-algebraic analog of Theorem 5.2 is well-known for the class of unital purely infinite simple $\mathrm{C}^{*}$-algebras, as a consequence of the powerful work of Kirchberg and Phillips, [11] and [16]. However, even in the concrete case of the subclass 
$\left\{\mathrm{M}_{d}\left(\mathcal{O}_{n}\right) \mid d, n \in \mathbb{N}\right\}$, the existence of the previously known isomorphisms in the $\mathrm{C}^{*}$-algebra setting (to wit, the aforementioned results of Rørdam, Kirchberg and Phillips) depend on deep results which produce no explicit isomorphisms. A natural question in this context is whether [16], Theorem 4.2.4, has an algebraic counterpart. In [3] the authors establish a partial affirmative answer to this question for a large class of purely infinite simple algebras.

\section{Graded isomorphisms Between LeavitT algebras and their matrix Rings.}

In this final section we incorporate the natural $\mathbb{Z}$-grading on the Leavitt algebras into our analysis. As one consequence, we will show that the sets of matrices which arise in the proof of [12, Theorem 5, cannot in general generate $\mathrm{M}_{d}\left(L_{n}\right)$.

The $\mathbb{Z}$-grading on $L_{K, n}$ is given as follows. We define the degree of a monomial of the form $y_{i}^{t} x_{j}^{u}$ by setting

$$
\operatorname{deg}\left(y_{i}^{t} x_{j}^{u}\right)=u-t
$$

and extending linearly to all of $L_{K, n}$. This is precisely the $\mathbb{Z}$-grading on $L_{K, n}$ induced by setting $\operatorname{deg}\left(X_{i}\right)=1, \operatorname{deg}\left(Y_{i}\right)=-1$ in $R=K<X_{1}, \ldots, X_{n}, Y_{1}, \ldots, Y_{n}>$, and then grading the factor ring $L_{n}=R / I$ in the natural way. (We note that the relations which define $L_{n}$ are homogeneous in this grading of $R$.)

It was shown in [2] that, in this grading, $\left(L_{n}\right)_{0} \cong \lim _{t \in \mathbb{N}}\left(\mathrm{M}_{n^{t}}(K)\right)$. Here the connecting homomorphisms are unital (so that the direct limit is unital); the homomorphism from $\mathrm{M}_{n^{t}}(K)$ to $\mathrm{M}_{n^{t+1}}(K)$ is given by sending any matrix of the form $\left(a_{i, j}\right)$ to the matrix $\left(a_{i, j} I_{n}\right)$.

We will need the following easily proved result about unital direct limits of rings. For a unital ring $R$, we say that a finite set $E=\left\{e_{1}, \ldots, e_{p}\right\}$ of idempotents in $R$ is complete, orthogonal, pairwise isomorphic in case $1_{R}=e_{1}+\ldots+e_{p}, e_{i} e_{j}=0$ for all $i \neq j$, and $R e_{i} \cong R e_{j}$ as left $R$-modules for all $1 \leq i, j \leq p$. In particular, in this situation we have $R \cong \oplus_{i=1}^{p} R e_{i}$ as left $R$-modules.

Lemma 6.1. Suppose $R$ is a unital direct limit of rings $R=\lim _{t \in \mathbb{N}}\left(R_{t}\right)$ (so we are assuming that connecting homomorphism $R_{t} \rightarrow R_{t+1}$ is unital for each $t \in \mathbb{N}$ ). Suppose $R$ contains a complete orthogonal pairwise isomorphic set of $p$ idempotents. Then there exists $m \in \mathbb{N}$ so that $R_{m}$ contains a complete orthogonal pairwise isomorphic set of $p$ idempotents.

Proof. Let $E=\left\{e_{1}, \ldots, e_{p}\right\}$ denote the indicated set in $R$. It is well known (see e.g. [10], Proposition III.7.4) that for idempotents $e$ and $f$ in any $\operatorname{ring} R, R e \cong R f$ as left $R$-modules if and only if there exist elements $x, y$ in $R$ such that $x=e x f, y=f y e, x y=e$, and $y x=f$. For each two-element subset $\left\{e_{i}, e_{j}\right\}$ of $E$ let $\left\{x_{i, j}, y_{i, j}\right\}$ denote a pair of associated elements whose existence is ensured by the supposed isomorphism $R e_{i} \cong R e_{j}$. Now pick $m \in \mathbb{N}$ with the property that $R_{m}$ contains the finite set $\left\{x_{i, j}, y_{i, j} \mid 1 \leq i, j \leq p\right\}$; such $m$ exists by definition of direct limit. Then necessarily $R_{m}$ contains $E$, as $x_{i, j} y_{i, j}=e_{i}$ for each $1 \leq i \leq p$. Now invoking the previously cited result from [10], and using the hypothesis that the direct limit has unital connecting homomorphisms, we conclude that $E$ is a complete orthogonal pairwise isomorphic set of $p$ idempotents in $R_{m}$.

Lemma 6.2. Let $S$ be any unital ring, let $K$ be a field, and let $p$ be any positive integer.

(1) If $p \mid d$, then the matrix ring $T=\mathrm{M}_{d}(S)$ contains a complete, orthogonal, pairwise isomorphic set of $p$ idempotents. 
(2) If the matrix ring $T=\mathrm{M}_{d}(K)$ contains a complete, orthogonal, pairwise isomorphic set of $p$ idempotents, then $p \mid d$.

Proof. For (1), writing $d=p q$ and using the isomorphism $\mathrm{M}_{d}(S) \cong \mathrm{M}_{p}\left(\mathrm{M}_{q}(S)\right)$ produces such a set, where we take $E$ to be the set of $p$ matrix idempotents in $\mathrm{M}_{p}\left(\mathrm{M}_{q}(S)\right)$.

For (2), let $E$ be such a set. The ring $T=\mathrm{M}_{d}(K)$ is semisimple artinian, with composition length $d$. As the left $T$-modules $T e_{i}$ generated by the elements of $E$ are pairwise isomorphic, each must have the same composition length, which we denote by $q$. But $T \cong \oplus_{i=1}^{p} T e_{i}$, which yields that $p q=d$.

With these two lemmas in hand, we are ready to prove the main result of this section.

Proposition 6.3. The algebras $L_{n}$ and $\mathrm{M}_{d}\left(L_{n}\right)$ are isomorphic as $\mathbb{Z}$-graded algebras if and only if there exists $\alpha \in \mathbb{N}$ such that $d \mid n^{\alpha}$.

Proof. First suppose there exists $\alpha \in \mathbb{N}$ such that $d \mid n^{\alpha}$. Then the explicit isomorphism provided in [14], Proposition 2.5, between the indicated matrix rings over Cuntz algebras is easily seen to restrict to an isomorphism of the analogously-sized matrix rings over Leavitt algebras. Furthermore, the isomorphism preserves the appropriate grading on these algebras, thus yielding the first implication. (For clarity, an explicit example of this isomorphism in a particular case is given below.)

Conversely, suppose the algebras $L_{n}$ and $\mathrm{M}_{d}\left(L_{n}\right)$ are isomorphic as $\mathbb{Z}$-graded algebras. Then necessarily the 0-components of these algebras are isomorphic. It is easy to show that the 0 component of $\mathrm{M}_{d}\left(L_{n}\right)$ is isomorphic to $\mathrm{M}_{d}\left(\lim _{t \in \mathbb{N}}\left(M_{n^{t}}(K)\right)\right)$. Now let $p$ be any prime number with $p \mid d$. Then by Lemma 6.2(1), $\mathrm{M}_{d}\left(\lim _{t \in \mathbb{N}}\left(M_{n^{t}}(K)\right)\right)$ contains a complete orthogonal pairwise isomorphic set of $p$ idempotents. Using the isomorphism between 0 -components, we get a complete orthogonal pairwise isomorphic set of $p$ idempotents in $\lim _{t \in \mathbb{N}}\left(\mathrm{M}_{n^{t}}(K)\right) \cong$ $\left(L_{n}\right)_{0}$. But by Lemma 6.1, this implies that there exists an integer $u$ so that the matrix ring $\mathrm{M}_{n^{u}}(K)$ contains a complete orthogonal pairwise isomorphic set of $p$ idempotents. By Lemma 6.2 (2) this implies that $p \mid n^{u}$, so that $p \mid n$ as $p$ is prime. Thus we have shown that any prime $p$ which divides $d$ also necessarily divides $n$, so that $d$ indeed divides some power of $n$ as desired.

Corollary 6.4. Suppose $\operatorname{gcd}(d, n-1)=1$. Suppose $W=\left\{X_{1}, \ldots, X_{n}, Y_{1}, \ldots, Y_{n}\right\}$ is a set of $2 n$ matrices in $\mathrm{M}_{d}\left(L_{n}\right)$ which satisfy the conditions of Proposition 1.1(2). Suppose further that each entry of $X_{i}$ (resp. $Y_{i}$ ) is either 0 or a monomial of degree 1 (resp. degree -1). If $W$ generates $\mathrm{M}_{d}\left(L_{n}\right)$ as a $K$-algebra, then $d \mid n^{\alpha}$ for some positive integer $\alpha$.

In particular, let $W$ be the set of $2 n$ matrices $\left\{X_{1}, \ldots, X_{n}, Y_{1}, \ldots, Y_{n}\right\}$ constructed in [12], Theorem 5. Then $W$ generates $\mathrm{M}_{d}\left(L_{n}\right)$ as a $K$-algebra if and only if $d \mid n^{\alpha}$ for some positive integer $\alpha$.

Proof. If $W$ satisfies the indicated conditions, then the homomorphism from $L_{n}$ to $\mathrm{M}_{d}\left(L_{n}\right)$ induced by the assignment $x_{i} \mapsto X_{i}$ and $y_{i} \mapsto Y_{i}$ in fact would be a graded isomorphism, and the result follows from Proposition 6.3.

In the specific case of the $2 n$ matrices described in [12, Theorem 5 , the matrices are of the indicated type, and were shown in [14] to generate $\mathrm{M}_{d}\left(L_{n}\right)$. 
It is instructive to compare and contrast the two types of generating sets of $\mathrm{M}_{d}\left(L_{n}\right)$ which can be constructed in case $d \mid n^{\alpha}$ for some $\alpha$. Let $d=3, n=6$. Here are the six matrices $\left\{X_{1}, \ldots, X_{6}\right\}$ which arise in the aforementioned construction presented in [14].

$$
\begin{aligned}
& X_{1}=\left(\begin{array}{ccc}
x_{1} & 0 & 0 \\
x_{2} & 0 & 0 \\
x_{3} & 0 & 0
\end{array}\right) \quad X_{2}=\left(\begin{array}{ccc}
x_{4} & 0 & 0 \\
x_{5} & 0 & 0 \\
x_{6} & 0 & 0
\end{array}\right) \quad X_{3}=\left(\begin{array}{ccc}
0 & x_{1} & 0 \\
0 & x_{2} & 0 \\
0 & x_{3} & 0
\end{array}\right) \\
& X_{4}=\left(\begin{array}{ccc}
0 & x_{4} & 0 \\
0 & x_{5} & 0 \\
0 & x_{6} & 0
\end{array}\right) \quad X_{5}=\left(\begin{array}{ccc}
0 & 0 & x_{1} \\
0 & 0 & x_{2} \\
0 & 0 & x_{3}
\end{array}\right) \quad X_{6}=\left(\begin{array}{ccc}
0 & 0 & x_{4} \\
0 & 0 & x_{5} \\
0 & 0 & x_{6}
\end{array}\right)
\end{aligned}
$$

In particular, all of these are of degree 1 in the $\mathbb{Z}$-grading, so that the assignment $x_{i} \mapsto X_{i}$ (and $x_{i}^{*} \mapsto X_{i}^{*}$ ) from $L_{6}$ to $\mathrm{M}_{3}\left(L_{6}\right)$ extends to a graded homomorphism, which can be shown in a straightforward way (using the argument given in [14]) to be a graded isomorphism.

In contrast, we now present one (of many) sets of generators for $\mathrm{M}_{3}\left(L_{6}\right)$ which arises from our construction. When $n=6, d=3$ then the appropriate data from our main result are as follows: $6=1 \cdot 3+3$, so $r=3, r-1=2, s=3-2=1, \hat{S}_{1}=\{1,2\}, \hat{S}_{2}=\{3\}, S_{1}=\{1,2,4,5\}$, $S_{2}=\{3,6\}$. So one possible collection of appropriate generating matrices in $\mathrm{M}_{3}\left(L_{6}\right)$ is

$$
\begin{array}{ccc}
X_{1}=\left(\begin{array}{ccc}
x_{1} & 0 & 0 \\
x_{2} & 0 & 0 \\
x_{3} & 0 & 0
\end{array}\right) & X_{2}=\left(\begin{array}{ccc}
x_{4} & 0 & 0 \\
x_{5} & 0 & 0 \\
x_{6} & 0 & 0
\end{array}\right) & X_{3}=\left(\begin{array}{ccc}
0 & 1 & 0 \\
0 & 0 & x_{1}^{2} \\
0 & 0 & x_{3} x_{1}
\end{array}\right) \\
X_{4}=\left(\begin{array}{lll}
0 & 0 & x_{2} x_{1} \\
0 & 0 & x_{4} x_{1} \\
0 & 0 & x_{6} x_{1}
\end{array}\right) & X_{5}=\left(\begin{array}{ccc}
0 & 0 & x_{5} x_{1} \\
0 & 0 & x_{2} \\
0 & 0 & x_{3}
\end{array}\right) & X_{6}=\left(\begin{array}{lll}
0 & 0 & x_{4} \\
0 & 0 & x_{5} \\
0 & 0 & x_{6}
\end{array}\right)
\end{array}
$$

We close this article by providing a brief historical perspective on this question. As mentioned earlier, Leavitt showed in [12] that if $R$ has module type $(1, n-1)$, then $\mathrm{M}_{d}(R)$ has module type $\left(1, \frac{n-1}{\operatorname{gcd}(d, n-1)}\right)$. The validity of this result is justified by the presentation of an appropriate set of elements inside $\mathrm{M}_{d}(R)$. In the situation where $R=L_{n}$ and $\operatorname{gcd}(d, n-1)=1$, it turns out that the appropriate set of elements inside $\mathrm{M}_{d}(R)$ is simply a lexicographic ordering of the variables $\left\{x_{1}, \ldots, x_{n}, y_{1}, \ldots, y_{n}\right\}$, using a straightforward algorithm. (An example of this process was given in Section 2, ) In the particular case when $d \mid n^{\alpha}$, the set of elements so constructed coincides with the set of elements analyzed by Paschke and Salinas in [14]; furthermore, this set just happens to generate all of $\mathrm{M}_{d}\left(L_{n}\right)$. However, as noted in Corollary 6.4, the analogous set of elements cannot generate all of $\mathrm{M}_{d}\left(L_{n}\right)$ when $d$ is not a divisor of some power of $n$. Thus, in order to establish our main result (Theorem 4.14), it was necessary to build a completely different set of tools than those which had already been used in this arena.

Corollary 6.4 shows that in general we cannot find generating sets of size $2 n$ inside $\mathrm{M}_{d}\left(L_{n}\right)$ in which each of the entries in the $n$ matrices has degree 1 (resp., each of the entries in the $n$ dual matrices has degree -1 ). In our main result we have shown that we can find generating sets of size $2 n$ inside $\mathrm{M}_{d}\left(L_{n}\right)$ in which each of the entries in the $n$ matrices has degree less than or equal to $d-1$ (resp., each of the entries in the $n$ dual matrices has degree greater than or equal to $1-d$ ). Reflecting on the examples given at the end of Section 4, it would be 
interesting to know whether in general it is possible to find generating sets of size $2 n$ inside $\mathrm{M}_{d}\left(L_{n}\right)$ in which each of the entries in the $n$ matrices has degree less than or equal to 2 (resp. each of the entries in the $n$ dual matrices has degree greater than or equal to -2 ).

\section{REFERENCES}

1. G. Abrams, Invariant basis number and types for strongly graded rings, J. Algebra 237 (2001), 32-37.

2. G. Abrams and P.N. Ánh, Some ultramatricial algebras which arise as intersections of Leavitt algebras, J. Alg. App. 1 (2002), no. 4, 357-363.

3. G. Abrams, P.N. Ánh and E. Pardo, Classification question for Leavitt path algebras, submitted.

4. G. Abrams and G. Aranda Pino, The Leavitt path algebra of a graph, J. Algebra 293 (2005), no. 2, 319-334.

5. G. Abrams and G. Aranda Pino, Purely infinite simple Leavitt path algebras, J. Pure Appl. Alg. 207 (2006), no. 3, 553-563.

6. P. Ara, K.R. Goodearl and E. Pardo, $K_{0}$ of purely infinite simple regular rings, K-Theory 26 (2002), 69-100.

7. G.M. Bergman, Coproducts and some universal ring constructions, Trans. A.M.S. 200 (1974), 33-88.

8. P.M. Cohn, Some remarks on the invariant basis property, Topology 5 (1966), 215 - 228.

9. J. Cuntz, Simple $\mathrm{C}^{*}$-algebras generated by isometries, Comm. Math. Physics 57 (1977), 173-185.

10. N. Jacobson, Structure of rings, American Mathematical Society Colloquium Publications Volume XXXVII, American Mathematical Society, Providence, R.I., 1956.

11. E. Kirchberg, The classification of purely infinite $\mathrm{C}^{*}$-algebras using Kasparov theory, preprint.

12. W.G. Leavitt, The module type of a ring, Transactions of the A.M.S. 103 (1962), 113-130.

13. W.G. Leavitt, The module type of homomorphic images, Duke Math. J. 32 (1965), 305-311.

14. W. Paschke and N. Salinas, Matrix algebras over $\mathcal{O}_{n}$, Michigan Math. J. 26 (1979), 3-12.

15. N.C. Phillips, Approximate unitary equivalence of homomorphisms from odd Cuntz algebras, in Operator algebras and their applications (Waterloo, ON, 1994/1995), 243-255. Fields Inst. Commun. 13, Amer. Math. Soc., Providence, RI, 1997.

16. N.C. Phillips, A classification theorem for nuclear purely infinite simple $\mathrm{C}^{*}$-algebras, Doc. Math. 5 (2000), 49-114.

17. I. Raeburn, Graph algebras, CBMS Regional Conference Series in Mathematics 103, Published for the Conference Board of the Mathematical Sciences, Washington, DC; by the American Mathematical Society, Providence, RI, 2005, vi + 113 pp.

18. M. Rørdam, Classification of inductive limits of Cuntz algebras, J. Reine Angew. Math. 440 (1993), 175-200.

19. J. Rosenberg, Algebraic K-Theory and its Applications, Graduate Texts in Mathematics 147, SpringerVerlag, New York, 1994, x + 392pp.

Department of Mathematics, University of Colorado, Colorado Springs CO 80933 U.S.A. E-mail address: abrams@math.uccs.edu

Rényi Institute of Mathematics, Hungarian Academy of Sciences, 1364 Budapest, Pf. 127 HUNGARY

E-mail address: anh@renyi.hu

Departamento de Matemáticas, Universidad de Cádiz, Apartado 40, 11510 Puerto Real (CÁdiz), Spain.

E-mail address: enrique.pardo@uca.es

URL: http://www2.uca.es/dept/matematicas/PPersonales/PardoEspino/index.HTML 